\title{
Aqueous Enantioselective Organocatalytic Diels-Alder Reactions Employing Hydrazide Catalysts. A New Scaffold for Organic Acceleration
}

\author{
Mathieu Lemay, William W. Ogilvie* \\ Department of Chemistry, University of Ottawa, 10 Marie Curie, Ottawa, Ontario Canada K1N \\ $6 N 5$ \\ wogilvie@science.uottawa.ca
}

\section{SUPPORTING INFORMATION}

General Experimental Remarks. All solvents were used as obtained from commercial suppliers unless otherwise indicated. Standard inert atmosphere techniques were employed in handling air and moisture sensitive reagents. All starting materials were purchased from Aldrich Chemical Company or Fluka and were used without purification unless otherwise stated.

Reactions were monitored by thin layer chromatography (TLC) using commercial aluminum-backed silica gel sheets coated with silica gel $60 \mathrm{~F}_{254}$ (E. Merck). TLC spots were visualized under ultraviolet light or developed by heating after treatment with potassium permanganate. Room temperature corresponds to $22{ }^{\circ} \mathrm{C}$. Excess solvents were removed in vacuo at pressures obtained by water or air aspirators connected to a Büchi rotary evaporator. Trace solvents were removed on a vacuum pump. Product purification by flash chromatography was performed with VWR Silica Gel 60 (230-400 mesh). Infrared (IR) spectra were obtained as neat films on a sodium chloride cell. All IR spectra were recorded on an ABB Bomem MB Series Fourier transform infrared spectrometer (FTIR). ${ }^{1} \mathrm{H}$ NMR (300 or $500 \mathrm{MHz}$ ) and ${ }^{13} \mathrm{C}$ NMR (75 or $125 \mathrm{MHz}$ ) were run on a Bruker AMX300 spectrometer, Bruker AMX500 spectrometer or Varian INOVA500 spectrometer. Chemical shifts are reported downfield from tetramethylsilane ( $\delta$ scale) in ppm. Mass spectroscopy (MS), using either electron impact (EI) or chemical ionization (CI), was performed on a V. G. Micromass 7070 HS mass spectrometer with an electron beam energy of $70 \mathrm{eV}$ (for EI). Electrospray analyses were run on an APCI/ESI triple quad mass spectrometer VG QUATTRO. High resolution mass spectroscopy (HRMS) was performed on a Kratos Concept-11A mass spectrometer with an electron beam of $70 \mathrm{eV}$, or an a JEOL double focusing magnetic sector mass spectrometer JMS-AX505H. GLC were performed on an Agilent 6890 Series gas chromatograph equipped with a split-mode capillary injection system and a flame ionization detector using Agilent/J\&W CycloSil-B column. Melting points were measured using a Melt Temp apparatus and are uncorrected. All mono-substituted 
hydrazines were prepared by syringe pump addition of a methanolic solution of alkyl or benzyl halides to an excess of hydrazine monohydrate $(10 \mathrm{eq})$ at $0{ }^{\circ} \mathrm{C} .{ }^{1}$

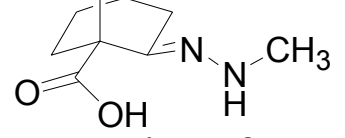

General procedure for the preparation of hydrazono-carboxylic acids. $(S)-(+)-7,7-$ Dimethyl-2-(methyl-hydrazono)-bicyclo[2.2.1]heptane-1-carboxylic acid.: Acetic acid (0.13 $\mathrm{mL}, 2.20 \mathrm{mmol})$ was added dropwise to a solution of $(S)-(+)-k^{-k}$ topinic $\operatorname{acid}^{2}(2.00 \mathrm{~g}, 11.0 \mathrm{mmol})$ and methylhydrazine $(758 \mathrm{mg}, 16.5 \mathrm{mmol})$ in anhydrous dichloromethane $(100 \mathrm{ml})$ at room temperature. The reaction mixture was stirred at that temperature until judged complete by TLC analysis (15 hours). Removal of solvent in vacuo and purification by silica gel chromatography $\left(5 \% \mathrm{MeOH}\right.$ in $\left.\mathrm{CHCl}_{3}\right)$ provided the compound as a white solid $(2.17 \mathrm{~g}, 94 \%)$. Mp 106-110 ${ }^{\circ} \mathrm{C}$; $[\alpha]_{\mathrm{D}}=+76.0\left(c 1.02, \mathrm{CHCl}_{3}\right)$; IR (neat) $3275,1720 \mathrm{~cm}^{-1} ;{ }^{1} \mathrm{H} \mathrm{NMR}\left(300 \mathrm{MHz}, \mathrm{CDCl}_{3}\right) \delta 8.80(\mathrm{br}$, 1H), $2.91(\mathrm{~s}, 3 \mathrm{H}), 2.42-2.33(\mathrm{~m}, 2 \mathrm{H})$ 2.1-1.9 (m, 3H), 1.82 (d, J=17.2 Hz, 1H), 1.71 (ddd, J=13.0, 9.3, $4.0 \mathrm{~Hz}, 1 \mathrm{H}) 1.33-1.23(\mathrm{~m}, 1 \mathrm{H}), 1.20(\mathrm{~s}, 3 \mathrm{H}), 0.86(\mathrm{~s}, 3 \mathrm{H}) ;{ }^{13} \mathrm{C} \mathrm{NMR}\left(75 \mathrm{MHz}, \mathrm{CDCl}_{3}\right) \delta$ $172.9(\mathrm{C}), 158.7(\mathrm{C}), 59.6(\mathrm{C}), 51.6(\mathrm{C}), 44.4(\mathrm{CH}), 38.0\left(\mathrm{CH}_{3}\right), 32.7\left(\mathrm{CH}_{2}\right), 32.3\left(\mathrm{CH}_{2}\right), 28.2$ $\left(\mathrm{CH}_{2}\right), 20.1\left(\mathrm{CH}_{3}\right) 19.8\left(\mathrm{CH}_{3}\right)$; MS (EI) $210.0\left(\mathrm{M}^{+}\right)$; HRMS (EI) calcd. for $\mathrm{C}_{11} \mathrm{H}_{18} \mathrm{~N}_{2} \mathrm{O}_{2}\left(\mathrm{M}^{+}\right)$ 210.1368; found 210.1369.

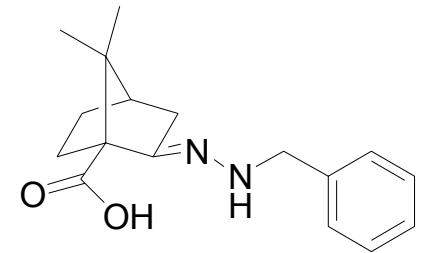

(S)-(+)-2-(Benzyl-hydrazono)-7,7-dimethyl-bicyclo[2.2.1]heptane-1-carboxylic acid. Prepared by a procedure similar to that described above from $(S)-(+)$-ketopinic acid ${ }^{2}(3.36 \mathrm{~g}$, $18.4 \mathrm{mmol})$ and benzylhydrazine $(3.38 \mathrm{~g}, 27.7 \mathrm{mmol})$. Purification by silica gel chromatography (30\% EtOAc in hexanes) provided the desired compound as a pale yellow oil (5.28 g, $100 \%$ ). $[\alpha]_{\mathrm{D}}=+53.7\left(c 1.10, \mathrm{CHCl}_{3}\right)$; IR (neat) $3282,1742 \mathrm{~cm}^{-1} ;{ }^{1} \mathrm{H}$ NMR $\left(500 \mathrm{MHz}, \mathrm{CDCl}_{3}\right) \delta 7.34-$ 7.24 (m, 5H), 4.31 (s, 2H), 2.39 (ddd, J=12.3, 12.1, 4.2 Hz, 1H), 2.32 (ddd J=17.1, 3.7, 3.7 Hz, $1 \mathrm{H}), 2.08-2.01(\mathrm{~m}, 1 \mathrm{H}), 1.97(\mathrm{t}, \mathrm{J}=4.38 \mathrm{~Hz}, 1 \mathrm{H}) 1.78(\mathrm{~d}, \mathrm{~J}=17.1 \mathrm{~Hz}, 1 \mathrm{H}), 1.72-1.67(\mathrm{~m}, 1 \mathrm{H})$,

\footnotetext{
${ }^{1}$ Finneman, J.; Fishbein, J. Am. Chem. Soc. 1995, 117, 4228-4239.

${ }^{2}$ Bartlett, P.P.; Knox, L.H. Organic Syntheses, Coll. Vol. 5, p.689; Vol. 45, p.55
} 
1.30-1.24 (m, 1H), $1.21(\mathrm{~s}, 3 \mathrm{H}), 0.79(\mathrm{~s}, 3 \mathrm{H}) ;{ }^{13} \mathrm{C}$ NMR (125 MHz, $\left.\mathrm{CDCl}_{3}\right) \delta 172.7(\mathrm{C}), 159.3$ $(\mathrm{C}), 138.2(\mathrm{C}), 128.6(\mathrm{CH}), 128.2(\mathrm{CH}), 127.6(\mathrm{CH}), 59.3(\mathrm{C}), 55.0\left(\mathrm{CH}_{2}\right), 51.4(\mathrm{C}), 44.3(\mathrm{CH})$, $32.6\left(\mathrm{CH}_{2}\right), 32.1\left(\mathrm{CH}_{2}\right), 28.1\left(\mathrm{CH}_{2}\right), 19.9\left(\mathrm{CH}_{3}\right), 19.6\left(\mathrm{CH}_{3}\right)$; MS (EI) $268.2\left(\mathrm{M}^{+}\right)$; HRMS (EI) calcd. for $\mathrm{C}_{17} \mathrm{H}_{22} \mathrm{~N}_{2} \mathrm{O}_{2}\left(\mathrm{M}^{+}\right) 286.1681$ found 286.1692 .

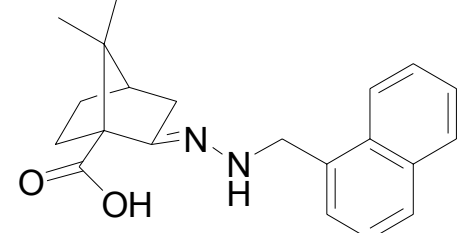

(S)-(+)-7,7-Dimethyl-2-(naphthalen-1-ylmethyl-hydrazono)-bicyclo [2.2.1]heptane-1carboxylic acid. Prepared by a procedure similar to that described above from $(S)-(+)$-ketopinic $\operatorname{acid}^{1}(1.97 \mathrm{~g}, 10.8 \mathrm{mmol})$ and naphtyl(1-methylhydrazine) $(2.25 \mathrm{~g}, 13.0 \mathrm{mmol})$. Purification by silica gel chromatography (30\% EtOAc in hexanes) provided the compound as a pale yellow oil (3.12 g, $86 \%$ ); IR (neat) 3278, $1724 \mathrm{~cm}^{-1} ;{ }^{1} \mathrm{H} \mathrm{NMR}\left(300 \mathrm{MHz}, \mathrm{CDCl}_{3}\right) \delta 8.08(\mathrm{~d}, \mathrm{~J}=8.4 \mathrm{~Hz}, 1 \mathrm{H})$, 7.88-7.77 (m, 2H), 7.57-7.39 (m, 4H), 4.82 (d, J=13.0 Hz, 1H), 4.76 (d, J=13.0 Hz, 1H), 2.39 (ddd, J=12.3, 12.3, 4.5 Hz, 1H), 2.25 (ddd, J=17.1, 3.7, 3.7 Hz, 1H), 2.08-1.88 (m, 2H), 1.74$1.63(\mathrm{~m}, 2 \mathrm{H}), 1.27-1.22(\mathrm{~m}, 1 \mathrm{H}), 1.20(\mathrm{~s}, 3 \mathrm{H}), 0.73(\mathrm{~s}, 3 \mathrm{H}) ;{ }^{13} \mathrm{C} \mathrm{NMR}\left(75 \mathrm{MHz}, \mathrm{CDCl}_{3}\right) \delta 172.8$ (C), 159.4 (C), 133.9 (C), 133.5 (C), $131.4(\mathrm{C}), 128.9(\mathrm{CH}), 128.6(\mathrm{CH}), 127.1(\mathrm{CH}), 126.4$ $(\mathrm{CH}), 125.9(\mathrm{CH}), 125.4(\mathrm{CH}), 123.3(\mathrm{CH}), 59.6(\mathrm{C}), 52.9\left(\mathrm{CH}_{2}\right), 51.5(\mathrm{C}), 44.3(\mathrm{CH}), 32.6$ $\left(\mathrm{CH}_{2}\right)$, $32.1\left(\mathrm{CH}_{2}\right), 28.2\left(\mathrm{CH}_{2}\right), 20.0\left(\mathrm{CH}_{3}\right), 19.6\left(\mathrm{CH}_{3}\right)$; MS (EI) $336.2\left(\mathrm{M}^{+}\right)$; HRMS (EI) calcd. for $\mathrm{C}_{21} \mathrm{H}_{24} \mathrm{~N}_{2} \mathrm{O}_{2}\left(\mathrm{M}^{+}\right) 336.1838$ found; 336.1822 .

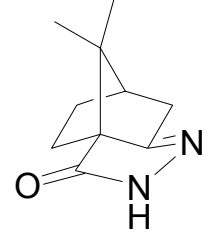

(S)-(+)-10,10-Dimethyl-3,4-diaza-tricyclo[5.2.1.0 $\left.{ }^{1,5}\right]$ dec-4-en-2-one: A solution of $(S)-(+)$-ketopinic $\operatorname{acid}^{2}(2.00 \mathrm{~g} 11.0 \mathrm{mmol})$ in $85 \%$ hydrazine hydrate $(10 \mathrm{~mL})$ was stirred at room temperature for 17 hours. Excess hydrazine and water were then removed from the white suspension by azeotropic distillation with toluene and the residue dried under vacuum for several hours. The white solid was suspended in mesitylene $(60 \mathrm{~mL})$ and the resulting mixture was refluxed while water was removed in dean-stark apparatus. Reflux was continued until consumption of the starting material was complete as judged by TLC. The cooled reaction mixture was directly loaded onto a silica column and was purified by flash chromatography 
(hexanes then with $50 \%$ EtOAc in hexanes) to afford a white solid (1.15 g, $59 \%$ ). Mp 176-178 ${ }^{\circ} \mathrm{C} ;[\alpha]_{\mathrm{D}}=+119\left(c 1.03, \mathrm{CHCl}_{3}\right)$; IR (neat) $3207,1678 \mathrm{~cm}^{-1} ;{ }^{1} \mathrm{H}$ NMR $\left(300 \mathrm{MHz}, \mathrm{CDCl}_{3}\right) \delta 8.91$ (br, $1 \mathrm{H}), 2.62-2.54(\mathrm{~m}, 1 \mathrm{H}), 2.29-2.2(\mathrm{~m}, 2 \mathrm{H}), 2.0-2.15(\mathrm{~m}, 2 \mathrm{H})$ 1.76-1.64 (m, $1 \mathrm{H}), 1.52-1.38(\mathrm{~m}$, 1H), 1.17 (s, 3H), 0.93 (s, 3H); ${ }^{13} \mathrm{C}$ NMR (75 MHz, $\left.\mathrm{CDCl}_{3}\right) \delta 176.7(\mathrm{C}), 175.6(\mathrm{C}), 62.4(\mathrm{C})$, $49.7(\mathrm{C}), 49.3(\mathrm{CH}), 32.0\left(\mathrm{CH}_{2}\right), 27.0\left(\mathrm{CH}_{2}\right), 25.3\left(\mathrm{CH}_{2}\right), 19.0\left(\mathrm{CH}_{3}\right), 18.5\left(\mathrm{CH}_{3}\right)$; $\mathrm{MS}(\mathrm{EI}) 178.1$ $\left(\mathrm{M}^{+}\right)$; HRMS (CI) calcd. for $\mathrm{C}_{10} \mathrm{H}_{14} \mathrm{~N}_{2} \mathrm{O}(\mathrm{M}+)$ 178.1106; found 178.1095 .

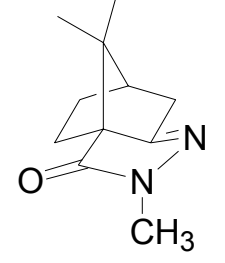

General procedure for the preparation of imino hydrazides. $(S)-(+)-3,10,10-$ Trimethyl-3,4-diaza-tricyclo[5.2.1.0 ${ }^{\mathbf{1 , 5}}$ ]dec-4-en-2-one. A solution of 7,7-Dimethyl-2(methyl-hydrazono)-bicyclo[2.2.1]heptane-1-carboxylic acid (800 mg, $3.80 \mathrm{mmol})$ in mesitylene $(38 \mathrm{~mL})$ was refluxed while water was removed in dean-stark apparatus. Reflux was continued until consumption of the starting material was complete as judged by TLC. The cooled reaction mixture was directly loaded onto a silica column and was purified by flash chromatography (hexanes followed by $30 \%$ EtOAc in hexanes) to provide the desired compound as a white solid (540 mg, $74 \%$ \%). $\mathrm{Mp} 87-88{ }^{\circ} \mathrm{C} ;[\alpha]_{\mathrm{D}}=+74.9$ (c 1.31, $\mathrm{CHCl}_{3}$ ); IR (neat) 1690, $1633 \mathrm{~cm}^{-1} ;{ }^{1} \mathrm{H}$ NMR (300MHz, $\left.\mathrm{CDCl}_{3}\right) \delta 3.23(\mathrm{~s}, 3 \mathrm{H}), 2.57-2.51(\mathrm{~m}, 1 \mathrm{H}), 1.96-2.79(\mathrm{~m}, 4 \mathrm{H}), 1.64-1.56(\mathrm{~m}$, $1 \mathrm{H}), 1.48-1.40(\mathrm{~m}, 1 \mathrm{H}), 1.14(\mathrm{~s}, 3 \mathrm{H}), 0.86(\mathrm{~s}, 3 \mathrm{H}) ;{ }^{13} \mathrm{C} \mathrm{NMR}\left(75 \mathrm{MHz}, \mathrm{CDCl}_{3}\right) \delta 174.2(\mathrm{C})$, $173.6(\mathrm{C}), 63.6(\mathrm{C}), 49.6(\mathrm{C}), 49.2(\mathrm{CH}), 31.8\left(\mathrm{CH}_{2}\right), 31.4\left(\mathrm{CH}_{3}\right), 26.9\left(\mathrm{CH}_{2}\right), 25.2\left(\mathrm{CH}_{2}\right), 19.1$ $\left(\mathrm{CH}_{3}\right), 18.6\left(\mathrm{CH}_{3}\right)$; MS (EI $192.1\left(\mathrm{M}^{+}\right)$; HRMS (EI) calcd. for $\mathrm{C}_{11} \mathrm{H}_{16} \mathrm{~N}_{2} \mathrm{O}(\mathrm{M}+)$ 192.1263; found 192.1252 .

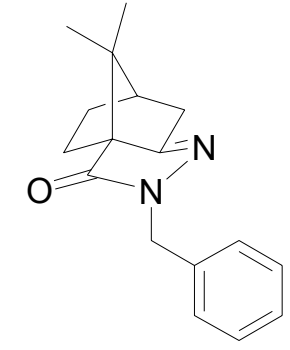

(S)-(+)-3-Benzyl-10,10-dimethyl-3,4-diaza-tricyclo[5.2.1.0 ${ }^{1,5}$ ]dec-4-en-2-one.

Prepared by a procedure similar to that described above for $(S)-(+)-10,10$-Dimethyl-3,4-diazatricyclo[5.2.1.0 $\left.{ }^{1,5}\right]$ dec-4-en-2-one from 2-(Benzyl-hydrazono)-7,7-dimethyl- 
bicyclo[2.2.1]heptane-1-carboxylic acid (3.43 g, $11.9 \mathrm{mmol})$. Purification by silica gel chromatography (hexanes then 30\% EtOAc in hexanes) provided the compound as a white solid (2.56 g, $80 \%$ ). Mp 90-92 ${ }^{\circ} \mathrm{C} ;[\alpha]_{\mathrm{D}}=+28.0\left(\mathrm{c} 1.04, \mathrm{CHCl}_{3}\right.$ ); IR (film) 1690, $1630 \mathrm{~cm}^{-1} ;{ }^{1} \mathrm{H} \mathrm{NMR}$ $\left(500 \mathrm{MHz}, \mathrm{CDCl}_{3}\right) \delta$ 7.30-7.20 (m, 5H), 4.79 (s, 2H), 2.53 (ddd, J=17.5, 3.6, 3.6 Hz, 1H), 2.26 (ddd, J=12.0, 4.3, 4.3 Hz, 1H), 2.21 (t, J=4.32 Hz, 1H), 2.13-2.06 (m, 2H), 1.64 (ddd, J=13.4, 9.5, $4.5 \mathrm{~Hz}, 1 \mathrm{H}), 1.45$ (ddd, J=13.3, 9.5, $4.3 \mathrm{~Hz}, 1 \mathrm{H}), 1.19$ (s, 3H), 0.87 (s, 3H); ${ }^{13} \mathrm{C}$ NMR $(125$ $\left.\mathrm{MHz}, \mathrm{CDCl}_{3}\right) \delta 174.4(\mathrm{C}), 173.5(\mathrm{C}), 137.1(\mathrm{C}), 128.4(\mathrm{CH}), 127.7(\mathrm{CH}), 127.3(\mathrm{CH}), 63.6(\mathrm{C})$, $49.7(\mathrm{C}), 49.1(\mathrm{CH}), 47.8\left(\mathrm{CH}_{2}\right), 31.8\left(\mathrm{CH}_{2}\right), 26.9\left(\mathrm{CH}_{2}\right), 25.2\left(\mathrm{CH}_{2}\right), 19.1\left(\mathrm{CH}_{3}\right), 18.5\left(\mathrm{CH}_{3}\right)$; MS (EI) 268.2 (M+); HRMS (EI) calcd.for $\mathrm{C}_{17} \mathrm{H}_{20} \mathrm{~N}_{2} \mathrm{O}(\mathrm{M}+)$ 268.1576; found 268.1589.

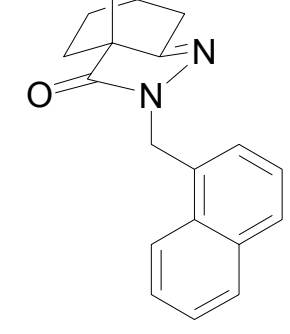

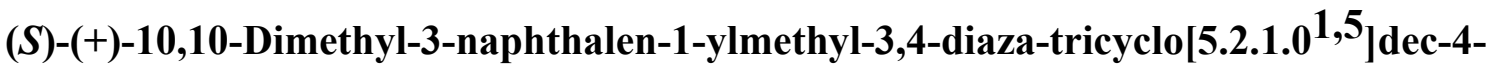

en-2-one. Prepared by a procedure similar to that described above for $(S)-(+)-10,10$-Dimethyl3,4-diaza-tricyclo[5.2.1.0 ${ }^{1,5}$ ]dec-4-en-2-one from 7,7-Dimethyl-2-(naphthalen-1-ylmethylhydrazono)-bicyclo[2.2.1]heptane-1-carboxylic acid (2.51 g, $7.88 \mathrm{mmol})$. Purification by silica gel chromatography (hexanes then $30 \%$ EtOAc in hexanes) provided the compound as a white solid (1.82 g, $72 \%)$. Mp 92-93 ${ }^{\circ} \mathrm{C} ;[\alpha]_{\mathrm{D}}+57.5\left(\mathrm{c} 1.10, \mathrm{CHCl}_{3}\right)$; IR (neat) $1690,1633 \mathrm{~cm}^{-1} ;{ }^{1} \mathrm{H}$ NMR (500 MHz, $\left.\mathrm{CDCl}_{3}\right) \delta 8.10(\mathrm{dd}, \mathrm{J}=5.1,0.3 \mathrm{~Hz}, 1 \mathrm{H}), 7.81(\mathrm{dd}, \mathrm{J}=14.4,4.8 \mathrm{~Hz}, 2 \mathrm{H}), 7.51-$ $7.41(\mathrm{~m}, 4 \mathrm{H}), 5.33(\mathrm{~d}, \mathrm{~J}=9.0 \mathrm{~Hz}, 1 \mathrm{H}), 5.14(\mathrm{~d}, \mathrm{~J}=9.0 \mathrm{~Hz}, 1 \mathrm{H}), 2.52-2.47(\mathrm{~m}, 1 \mathrm{H}), 2.28$ (ddd, J = 7.2, 7.2, $2.7 \mathrm{~Hz}, 1 \mathrm{H}), 2.19(\mathrm{dd}, \mathrm{J}=2.4,2.4 \mathrm{~Hz}, 1 \mathrm{H}), 2.12-2.02(\mathrm{~m}, 1 \mathrm{H}), 2.04(\mathrm{~d}, \mathrm{~J}=10.5 \mathrm{~Hz}, 1 \mathrm{H})$, 1.57 (ddd, J = 8.1, 5.7, $2.7 \mathrm{~Hz}, 1 \mathrm{H}), 1.41$ (ddd, J = 7.8, 5.7, $2.7 \mathrm{~Hz}, 1 \mathrm{H}), 1.22$ (s, 3H), 0.84 (s, $3 \mathrm{H}) ;{ }^{13} \mathrm{C}$ NMR (125 MHz, $\left.\mathrm{CDCl}_{3}\right) \delta 174.6(\mathrm{C}), 173.1(\mathrm{C}), 133.7$ (C), $132.5(\mathrm{C}), 131.2(\mathrm{C}), 128.5$ $(\mathrm{CH}), 128.5(\mathrm{CH}), 127.4(\mathrm{CH}), 126.2(\mathrm{CH}), 125.7(\mathrm{CH}), 125.2(\mathrm{CH}), 123.7(\mathrm{CH}), 63.7(\mathrm{C}), 49.7$ (C), $49.1(\mathrm{CH}), 46.1\left(\mathrm{CH}_{2}\right), 31.9\left(\mathrm{CH}_{2}\right), 26.8\left(\mathrm{CH}_{2}\right), 25.3\left(\mathrm{CH}_{2}\right), 19.1\left(\mathrm{CH}_{3}\right), 18.5\left(\mathrm{CH}_{3}\right) ; \mathrm{MS}$ $318.2\left(\mathrm{M}^{+}\right)$; HRMS calcd. for $\mathrm{C}_{21} \mathrm{H}_{22} \mathrm{~N}_{2} \mathrm{O}(\mathrm{M}+)$ 318.1732; found 318.1737 . 


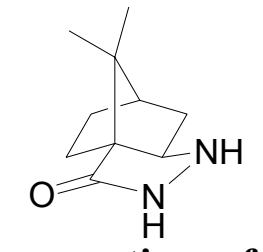

General procedure for the preparation of catalysts. $(S)-(+)-10,10-D i m e t h y l-3,4-$ diaza-tricyclo[5.2.1.0 ${ }^{1,5}$ ]decan-2-one (8): To a solution of 10,10-Dimethyl-3,4-diazatricyclo[5.2.1.0 $\left.{ }^{1,5}\right]$ dec-4-en-2-one $(1.05 \mathrm{~g}, 5.89 \mathrm{mmol})$ in a $2: 1$ mixture of acetic acid and methanol $(60 \mathrm{~mL})$ was added sodium cyanoborohydride $(3.70 \mathrm{~g}, 58.9 \mathrm{mmol})$ in small portions over $1 \mathrm{~h}$. The reaction mixture was then stirred at room temperature until TLC indicated that the reaction was complete (16 hours). Excess borohydride was quenched by the addition of $10 \%$ $\mathrm{HCl}$. The products were extracted using $\mathrm{CH}_{2} \mathrm{Cl}_{2}$ and the aqueous phase was made basic using sodium hydroxide pellets then further extracted with $\mathrm{CH}_{2} \mathrm{Cl}_{2}$. The organic extracts were combined, washed with brine and dried over sodium sulphate. The solvent was removed in vacuo and the product was purified by flash chromatography $\left(10 \% \mathrm{MeOH}\right.$ in $\left.\mathrm{CHCl}_{3}\right)$ to afford the desired compound as a white solid $(914 \mathrm{mg}, 86 \%)$. Mp 206-209 ${ }^{\circ} \mathrm{C}$; $[\alpha]_{\mathrm{D}}=+33.9(c$ 1.04, $\mathrm{CHCl}_{3}$ ); IR (neat) 3427, $1659 \mathrm{~cm}^{-1} ;{ }^{1} \mathrm{H}$ NMR $\left(300 \mathrm{MHz}, \mathrm{CDCl}_{3}\right) \delta 5.76(\mathrm{br}, 1 \mathrm{H}), 3.73$ (dd, J=8.4, $4.7 \mathrm{~Hz}, 1 \mathrm{H}) 2.17-2.05(\mathrm{~m}, 2 \mathrm{H}) 1.98-1.84(\mathrm{~m}, 2 \mathrm{H}) 1.72(\mathrm{dd}, \mathrm{J}=13.1,8.4 \mathrm{~Hz}, 1 \mathrm{H}) 1.34-1.19(\mathrm{~m}$, 2H), $1.27(\mathrm{~s}, 3 \mathrm{H}), 1.06(\mathrm{~s}, 3 \mathrm{H}) ;{ }^{13} \mathrm{C} \mathrm{NMR}\left(75 \mathrm{MHz}, \mathrm{CDCl}_{3}\right) \delta 175.6(\mathrm{C}), 69.0(\mathrm{CH}), 57.6(\mathrm{C})$, $50.6(\mathrm{C}), 46.9(\mathrm{CH}), 36.4\left(\mathrm{CH}_{2}\right), 28.3\left(\mathrm{CH}_{2}\right), 26.6\left(\mathrm{CH}_{2}\right), 21.0\left(\mathrm{CH}_{3}\right), 20.4\left(\mathrm{CH}_{3}\right)$; $\mathrm{MS}(\mathrm{EI}) 180.1$ $\left(\mathrm{M}^{+}\right)$; HRMS (EI) calcd. for $\mathrm{C}_{10} \mathrm{H}_{16} \mathrm{~N}_{2} \mathrm{O}(\mathrm{M}+)$ 180.1263; found 180.1248 .

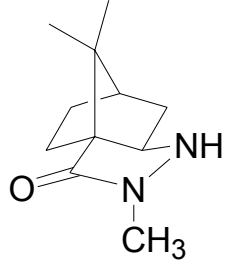

(S)-(+)-3,10,10-Trimethyl-3,4-diaza-tricyclo[5.2.1.0 ${ }^{1,5}$ ]decan-2-one (9). Prepared by a procedure similar to that described above for $\mathbf{8}$ from 3,10,10-Trimethyl-3,4-diazatricyclo[5.2.1.0 ${ }^{1,5}$ ]dec-4-en-2-one $(450 \mathrm{mg}, 2.34 \mathrm{mmol})$. Purification by silica gel chromatography (50\% EtOAc in hexanes) provided the title compound as a white solid (434 mg, $95 \%$ ). Mp 101-104 ${ }^{\circ} \mathrm{C} ;[\alpha]_{\mathrm{D}}=+33.3\left(c 1.01, \mathrm{CHCl}_{3}\right)$; IR (neat) $3431,1656 \mathrm{~cm}^{-1} ;{ }^{1} \mathrm{H}$ NMR (300 $\left.\mathrm{MHz}, \mathrm{CDCl}_{3}\right) \delta 4.10(\mathrm{br}, 1 \mathrm{H}), 3.57(\mathrm{dd}, \mathrm{J}=8.4,4.7 \mathrm{~Hz}, 1 \mathrm{H}) 2.98(\mathrm{~s}, 3 \mathrm{H}) 2.17-2.03(\mathrm{~m}, 2 \mathrm{H})$ 1.96$1.83(\mathrm{~m}, 2 \mathrm{H}) 1.69(\mathrm{dd}, \mathrm{J}=13.0,8.4 \mathrm{~Hz}, 1 \mathrm{H}) 1.30-1.18(\mathrm{~m}, 2 \mathrm{H}), 1.15(\mathrm{~s}, 3 \mathrm{H}), 1.05(\mathrm{~s}, 3 \mathrm{H}) ;{ }^{13} \mathrm{C}$ 
NMR (75 MHz, $\left.\mathrm{CDCl}_{3}\right) \delta 170.9(\mathrm{C}), 65.2(\mathrm{CH}), 58.6(\mathrm{C}), 51.2(\mathrm{C}), 46.7(\mathrm{CH}), 36.6\left(\mathrm{CH}_{2}\right), 31.0$ $\left(\mathrm{CH}_{3}\right), 28.5\left(\mathrm{CH}_{2}\right), 26.7\left(\mathrm{CH}_{2}\right), 20.9\left(\mathrm{CH}_{3}\right), 20.3\left(\mathrm{CH}_{3}\right)$; MS $194.1\left(\mathrm{M}^{+}\right)$; HRMS (EI) calcd. for $\mathrm{C}_{11} \mathrm{H}_{18} \mathrm{~N}_{2} \mathrm{O}\left(\mathrm{M}^{+}\right)$194.1419; found 194.1430.

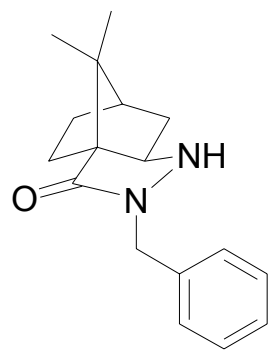

\section{(S)-(+)-3-Benzyl-10,10-dimethyl-3,4-diaza-tricyclo[5.2.1.0 1,5 decan-2-one}

(5).

Prepared by a procedure similar to that described above for 8 from 3-Benzyl-10,10-dimethyl-3,4diaza-tricyclo[5.2.1.0 ${ }^{1,5}$ ]dec-4-en-2-one $(2.40 \mathrm{~g}, 8.94 \mathrm{mmol})$. Purification by silica gel chromatography (30\% EtOAc in hexanes) provided the desired compound as a white solid (2.28 g, $94 \%$ ). Mp 113-115 ${ }^{\circ} \mathrm{C} ;[\alpha]_{\mathrm{D}}=+12.6\left(c 1.03, \mathrm{CHCl}_{3}\right)$; IR (neat) 3210, $1656 \mathrm{~cm}^{-1} ;{ }^{1} \mathrm{H}$ NMR $\left(500 \mathrm{MHz}, \mathrm{CDCl}_{3}\right) \delta$ 7.30-7.22 (m, 5H), 4.68 (d, J=14.3 Hz, 1H), 4.41 (d, J=14.0 Hz, 1H), 3.48 (dd, J=8.3, 4.7 Hz, 1H) 2.14 (dt, J=11.6, $4.8 \mathrm{~Hz}, 1 \mathrm{H}), 1.97$ (ddd, J=12.9, 7.6, 3.4 Hz, 1H), 1.90$1.82(\mathrm{~m}, 2 \mathrm{H}), 1.62$ (dd, J=13.1, 8.4 Hz, 1H), 1.27 (ddd, J=11.9, 9.1, 2.7 Hz, 1H), 1.22-1.17 (m, 1H), 1.08 (s, 3H), 1.05 (s, 3H); ${ }^{13} \mathrm{C}$ NMR (125 MHz, $\left.\mathrm{CDCl}_{3}\right) \delta 170.6(\mathrm{C}), 135.9(\mathrm{C}), 128.6(\mathrm{CH})$, $128.3(\mathrm{CH}), 127.7(\mathrm{CH}), 65.2(\mathrm{CH}), 58.3(\mathrm{C}), 51.1(\mathrm{C}), 47.9\left(\mathrm{CH}_{2}\right), 46.7(\mathrm{CH}), 36.3\left(\mathrm{CH}_{2}\right), 28.6$ $\left(\mathrm{CH}_{2}\right), 26.6\left(\mathrm{CH}_{2}\right), 20.9\left(\mathrm{CH}_{3}\right), 20.2\left(\mathrm{CH}_{3}\right)$; $\mathrm{MS}(\mathrm{EI}) 270.2\left(\mathrm{M}^{+}\right)$; HRMS (EI) calcd. for $\mathrm{C}_{17} \mathrm{H}_{22} \mathrm{~N}_{2} \mathrm{O}(\mathrm{M}+)$ 270.1732; found 270.1723.

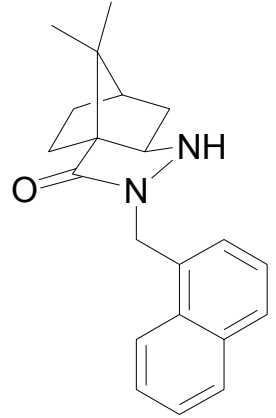

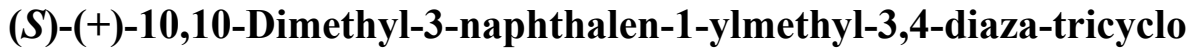

[5.2.1.0 $\left.{ }^{1,5}\right]$ decan-2-one (10). Prepared by a procedure similar to that described above for 8 from 10-Dimethyl-3-naphthalen-1-ylmethyl-3,4-diaza-tricyclo[5.2.1.0 1,5 $]$ dec-4-en-2-one (1.60 g, 5.02 mmol). Purification by silica gel chromatography $(30 \%$ EtOAc in hexanes provided $\mathbf{1 0}$ as a 
white solid (1.43 g, $89 \%) \mathrm{mp} 144-145{ }^{\circ} \mathrm{C} ;[\alpha]_{\mathrm{D}}+39.5\left(\mathrm{c}=1.00, \mathrm{CHCl}_{3}\right)$; IR (neat) 3245,1667 $\mathrm{cm}^{-1} ;{ }^{1} \mathrm{H}$ NMR $\left(500 \mathrm{MHz}, \mathrm{CDCl}_{3}\right) \delta 8.27(\mathrm{~d}, \mathrm{~J}=4.8 \mathrm{~Hz}, 1 \mathrm{H}), 7.81(\mathrm{dd}, \mathrm{J}=10.2,4.8 \mathrm{~Hz}, 2 \mathrm{H})$, 7.56-7.37 (m, 4H), 5.26 (br, 1H), 4.78 (br, 1H), 3.88 (br, 1H), 3.43-3.40 (m, 1H), 2.17 (ddd, J = 7.2, 7.2, 3.0 Hz, 1H), 1.97-1.81 (m, 3H), $1.58(\mathrm{dd}, \mathrm{J}=7.5,4.8 \mathrm{~Hz}, 1 \mathrm{H}), 1.28-1.15$ (m, $2 \mathrm{H}), 1.08$ (s, 3H), 1.02 (s, 3H); ${ }^{13} \mathrm{C}$ NMR (125 MHz, $\left.\mathrm{CDCl}_{3}\right) \delta 170.0(\mathrm{C}), 133.7$ (C), 131.4 (C), 128.9 $(\mathrm{CH}), 128.4(\mathrm{CH}), 127.9(\mathrm{CH}), 126.6(\mathrm{CH}), 126.0(\mathrm{CH}), 125.0(\mathrm{CH}), 124.1(\mathrm{CH}), 64.9(\mathrm{CH})$, $58.6(\mathrm{C}), 51.3(\mathrm{C}), 46.6(\mathrm{CH}), 46.2\left(\mathrm{CH}_{2}\right), 36.4\left(\mathrm{CH}_{2}\right), 28.5\left(\mathrm{CH}_{2}\right), 26.6\left(\mathrm{CH}_{2}\right), 20.8\left(\mathrm{CH}_{3}\right), 20.1$ $\left(\mathrm{CH}_{3}\right)$; MS 320.2 (M $\left.\mathrm{M}^{+}\right)$; HRMS calcd for $\mathrm{C}_{21} \mathrm{H}_{24} \mathrm{~N}_{2} \mathrm{O} 320.1889$; found 320.1894 .

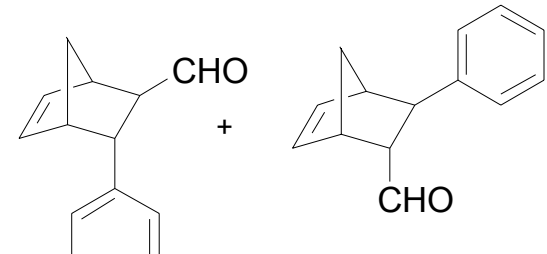

General Procedure for Hydrazide-Catalyzed Diels-Alder Reactions. $(1 R, 2 R, 3 R$, 4S)-3-phenylbicyclo[2.2.1] hept-5-ene-2-carboxaldehyde and $(1 S, \quad 2 S, \quad 3 S, \quad 4 R)-3-$ phenylbicyclo[2.2.1]hept-5-ene-2-carboxaldehyde (Table 3, entry 1): To a suspension of $(E)$ cinnamaldehyde $(500 \mathrm{mg}, 3.78 \mathrm{mmol})$ in distilled water $(3.8 \mathrm{ml})$ was added 3-Benzyl-10,10dimethyl-3,4-diaza-tricyclo[5.2.1.0 ${ }^{1,5}$ ]decan-2-one $5(204 \mathrm{mg}, 0.756 \mathrm{mmol})$ followed by $\mathrm{CF}_{3} \mathrm{SO}_{3} \mathrm{H}(67 \mu \mathrm{L}, 0.76 \mathrm{mmol})$. After stirring for 1 to 2 minutes cyclopentadiene (749 mg, 11.3 mmol) was slowly added and the resulting mixture was stirred at room temperature until the reaction was judged to be complete by TLC analysis. The reaction mixture was extracted twice with ether and the combined organic extracts were washed successively with water and brine then dried over $\mathrm{Na}_{2} \mathrm{SO}_{4}$. Purification by silica gel chromatography (5\% EtOAc in hexanes) provided the desired material as a 1.9:1 mixture of exo and endo isomers (colourless oil, $721 \mathrm{mg}$, $96 \%$ ). Exo ee $90 \%$, endo ee $88 \%$. Enantiomeric ratios were determined using chiral GLC analysis (Agilent/J\&W CycloSil-B, $100{ }^{\circ} \mathrm{C}$ hold 3 min then $2{ }^{\circ} \mathrm{C} / \mathrm{min}$ gradient, flow $=3.0$ $\mathrm{mL} / \mathrm{min}$ ) exo isomers $\mathrm{t}_{\mathrm{r}}=42.7 \mathrm{~min}, 43.8 \mathrm{~min}$, endo isomers $\mathrm{t}_{\mathrm{r}}=43.4 \mathrm{~min}, 44.3 \mathrm{~min}$. The same enantiomeric ratios were obtained by acetalization with $(+)-(R, R)$-hydrobenzoin and ${ }^{1} \mathrm{H}$ NMR analysis: ${ }^{3}\left(500 \mathrm{MHz}, \mathrm{C}_{6} \mathrm{D}_{6}\right.$ ) exo isomers $\delta 5.57$ (d, $\mathrm{J}=4.8 \mathrm{~Hz}, \mathrm{CHO}_{2}$, major isomer), endo isomers $\delta 5.21\left(\mathrm{~d}, \mathrm{~J}=8.1 \mathrm{~Hz}, \mathrm{CHO}_{2}\right.$, major isomer), 5.56 (d, J=4.8 Hz, $\mathrm{CHO}_{2}$, minor isomer), 5.17 (d,

\footnotetext{
${ }^{3}$ Fujioka, H.; Kotoku, N.; Fujita, T.; Inoguchi, R.; Murai, K; Nagatomi, Y.; Sawama, Y.; Kita, Y. Chirality, 2003, 15,60 .
} 
$\mathrm{J}=8.2 \mathrm{~Hz}, \mathrm{CHO}_{2}$, minor isomer). ${ }^{1} \mathrm{H}$ NMR, ${ }^{13} \mathrm{C}$ NMR and IR data were identical to those previously reported. ${ }^{4}$

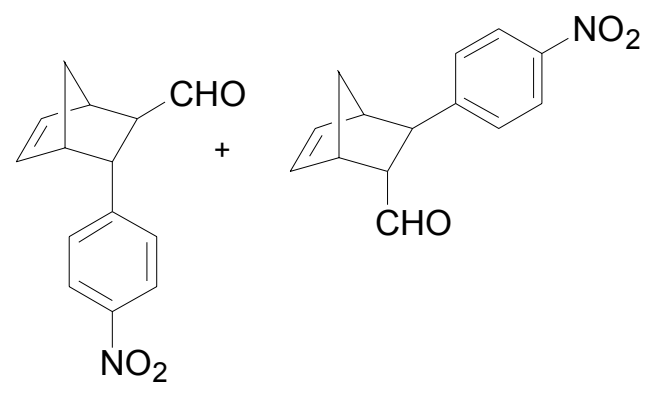

$(1 R, 2 R, 3 R, 4 S)$-3-(4-Nitrophenyl)-bicyclo[2.2.1] hept-5-ene-2-carboxaldehyde and (1S, 2S, 3S, 4R)-3-(4-Nitrophenyl)-bicyclo[2.2.1]hept-5-ene-2-carboxaldehyde (Table 3, entry 2). Prepared according to the general procedure described above from $(E)-4-$ nitrocinnamaldehyde (100 mg, $0.564 \mathrm{mmol})$ and cyclopentadiene (112 mg, $1.69 \mathrm{mmol})$. Purification by silica gel chromatography (15\% EtOAc in hexanes) provided the desired material as a 2.2:1 mixture of exo and endo isomers (pale yellow oil, $128 \mathrm{mg}, 93 \%$ ). Exo ee $92 \%$, endo ee $87 \%$. Enantiomeric ratios were determined by acetalization with $(+)-(R, R)$-hydrobenzoin and ${ }^{1} \mathrm{H}$ NMR analysis: (500 MHz, $\mathrm{C}_{6} \mathrm{D}_{6}$ ) exo isomers $\delta 5.48$ (d, $\mathrm{J}=5.2 \mathrm{~Hz}, \mathrm{CHO}_{2}$, major isomer), 5.46 (d, J=6.0 Hz, $\mathrm{CHO}_{2}$, minor isomer), endo isomers $\delta 5.14$ (d, J=8.1 Hz, $\mathrm{CHO}_{2}$, major isomer), 5.07 (d, J=8.2 Hz, $\mathrm{CHO}_{2}$, minor isomer). [ $\left.\alpha\right]_{\mathrm{D}}-198$ (c $1.11 \mathrm{CHCl}_{3}$ ); IR (neat) 1720, 1591, 1511, $1344 \mathrm{~cm}^{-1}$; exo isomer ${ }^{1} \mathrm{H}$ NMR $\left(300 \mathrm{MHz}, \mathrm{CDCl}_{3}\right) \delta 9.87(\mathrm{~d}, \mathrm{~J}=1.5 \mathrm{~Hz}, 1 \mathrm{H}), 8.06-8.01(\mathrm{~m}$, 2H), $7.25(\mathrm{dd}, \mathrm{J}=8.4,0.6 \mathrm{~Hz}, 2 \mathrm{H}), 6.36(\mathrm{dd}, \mathrm{J}=5.7,3.3 \mathrm{~Hz}, 1 \mathrm{H}), 6.00(\mathrm{dd}, \mathrm{J}=5.7,2.7 \mathrm{~Hz}, 1 \mathrm{H})$, $3.84(\mathrm{dd}, \mathrm{J}=4.5,4.5 \mathrm{~Hz}, 1 \mathrm{H}), 3.26-3.13(\mathrm{~m}, 2 \mathrm{H}), 2.59$ (dd, J = 5.1, $0.6 \mathrm{~Hz}, 1 \mathrm{H}), 1.74-1.63(\mathrm{~m}$, 2H); ${ }^{13} \mathrm{C}$ NMR (75 MHz, CDCl $) \delta 202.0(\mathrm{CH}), 151.0(\mathrm{C}), 146.8(\mathrm{C}), 137.4(\mathrm{CH}), 136.3(\mathrm{CH})$, 129.1 (CH), $123.7(\mathrm{CH}), 59.9(\mathrm{CH}), 48.8(\mathrm{CH}), 48.0\left(\mathrm{CH}_{2}\right), 45.9(\mathrm{CH}), 45.5(\mathrm{CH})$; endo isomer ${ }^{1} \mathrm{H}$ NMR $\left(300 \mathrm{MHz}, \mathrm{CDCl}_{3}\right) \delta 9.60(\mathrm{~d}, \mathrm{~J}=1.5 \mathrm{~Hz}, 1 \mathrm{H}), 8.11-8.08(\mathrm{~m}, 2 \mathrm{H}), 7.38(\mathrm{dd}, \mathrm{J}=8.4,0.6$ Hz, 2H), 6.39 (dd, J = 5.7, 3.3 Hz, 1H), 6.15 (dd, J = 5.7, 3.0 Hz, 1H), 3.39 (br, 1H), 3.26-3.13 (m, 2H), 2.94-2.90 (m, 1H), 1.74-1.63 (m, 2H); $\left.{ }^{13} \mathrm{C} \mathrm{NMR} \mathrm{(75} \mathrm{MHz,} \mathrm{CDCl}_{3}\right) \delta 202.6(\mathrm{CH}), 152.0$ (C), $146.7(\mathrm{C}), 139.4(\mathrm{CH}), 134.3(\mathrm{CH}), 128.6(\mathrm{CH}), 124.1(\mathrm{CH}), 61.5(\mathrm{CH}), 48.3(\mathrm{CH}), 47.5$ $\left(\mathrm{CH}_{2}\right), 45.9(\mathrm{CH}), 45.4(\mathrm{CH})$. MS (ESI) $\left(\mathrm{MH}^{+}\right) 244.1$.

\footnotetext{
${ }^{4}$ Ishihara, K.; Kuruhara, H; Matsumoto, M.; Yamamoto, H. J. Am. Chem. Soc. 1998, 120, 6920.
} 


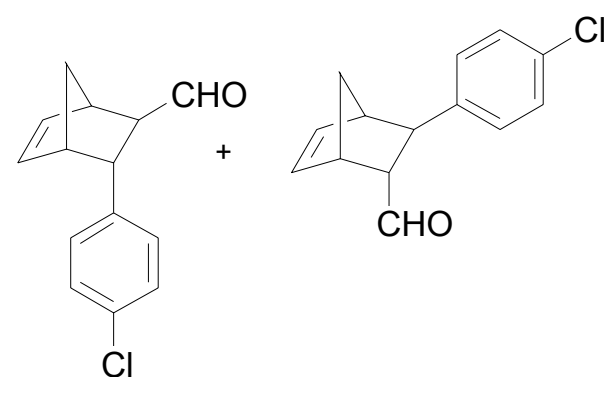

$(1 R, 2 R, 3 R, 4 S)$-3-(4-chlorophenyl)-bicyclo[2.2.1] hept-5-ene-2-carboxaldehyde and $(1 S, 2 S, 3 S, 4 R)$-3-(4-Chlorophenyl)-bicyclo[2.2.1] hept-5-ene-2-carboxaldehyde (Table 3, entry 3). Prepared according to the general procedure described above from $(E)-4-$ chlorocinnamaldehyde $(125 \mathrm{mg}, 0.750 \mathrm{mmol})$ and cyclopentadiene (149 $\mathrm{mg}, 2.25 \mathrm{mmol})$. Purification by silica gel chromatography (5\% EtOAc in hexanes) provided the desired material as a 2:1 mixture of exo and endo isomers (colourless oil, $161 \mathrm{mg}, 92 \%$ ). Exo ee $87 \%$, endo ee $90 \%$. Enantiomeric ratios were determined by acetalization with $(+)-(R, R)$-hydrobenzoin and ${ }^{1} \mathrm{H}$ NMR analysis: (500 MHz, $\mathrm{C}_{6} \mathrm{D}_{6}$ ) exo isomers $\delta 5.54$ (d, J=5.0 Hz, $\mathrm{CHO}_{2}$, major isomer), 5.52 (d, $\mathrm{J}=5.9 \mathrm{~Hz}, \mathrm{CHO}_{2}$, minor isomer), endo isomers $\delta 5.19$ (d, J=8.2 Hz, $\mathrm{CHO}_{2}$, major isomer), 5.13 (d, J=8.3 Hz, $\mathrm{CHO}_{2}$, minor isomer). $[\alpha]_{\mathrm{D}}-181.0\left(\right.$ c $\left.1.07 \mathrm{CHCl}_{3}\right)$; IR (neat) $1713 \mathrm{~cm}^{-1}$; exo isomer ${ }^{1} \mathrm{H}$ NMR $\left(300 \mathrm{MHz}, \mathrm{CDCl}_{3}\right) \delta 9.87(\mathrm{~d}, \mathrm{~J}=2.1 \mathrm{~Hz}, 1 \mathrm{H}), 7.23-7.15(\mathrm{~m}, 2 \mathrm{H}), 7.44(\mathrm{~d}, \mathrm{~J}=8.4 \mathrm{~Hz}$, 2H), $6.32(\mathrm{dd}, \mathrm{J}=5.7,3.0 \mathrm{~Hz}, 1 \mathrm{H}), 6.01(\mathrm{dd}, \mathrm{J}=5.7,3.0 \mathrm{~Hz}, 1 \mathrm{H}), 3.68(\mathrm{dd}, \mathrm{J}=4.8,3.6 \mathrm{~Hz}, 1 \mathrm{H})$, 3.20-3.15 (m, 1H), 3.07-3.02 (m, 1H), 2.52-2.50 (m, 1H), 1.55-1.51 (m, 2H); ${ }^{13} \mathrm{C}$ NMR $(75$ $\left.\mathrm{MHz}, \mathrm{CDCl}_{3}\right) \delta 202.7(\mathrm{CH}), 141.5(\mathrm{C}), 136.9(\mathrm{CH}), 136.7(\mathrm{CH}), 132.4(\mathrm{C}), 129.6(\mathrm{CH}), 128.6$ $(\mathrm{CH}), 60.0(\mathrm{CH}), 48.8(\mathrm{CH}), 47.9\left(\mathrm{CH}_{2}\right), 45.8(\mathrm{CH}), 45.1(\mathrm{CH})$; endo ${ }^{1} \mathrm{H}$ NMR $(300 \mathrm{MHz}$, $\left.\mathrm{CDCl}_{3}\right) \delta 9.56(\mathrm{~d}, \mathrm{~J}=1.8 \mathrm{~Hz}, 1 \mathrm{H}), 7.26-7.15(\mathrm{~m}, 4 \mathrm{H}), 6.38(\mathrm{dd}, \mathrm{J}=5.7,3.3 \mathrm{~Hz}, 1 \mathrm{H}), 6.14(\mathrm{dd}, \mathrm{J}$ $=5.7,3.0 \mathrm{~Hz}, 1 \mathrm{H}), 3.32(\mathrm{br}, 1 \mathrm{H}), 3.20-3.15(\mathrm{~m}, 2 \mathrm{H}), 2.90-2.87(\mathrm{~m}, 1 \mathrm{H}), 1.62-1.58(\mathrm{~m}, 1 \mathrm{H}), 1.55-$ $1.51(\mathrm{~m}, 1 \mathrm{H}) ;{ }^{13} \mathrm{C}$ NMR $\left(75 \mathrm{MHz}, \mathrm{CDCl}_{3}\right) \delta 203.4(\mathrm{CH}), 142.5(\mathrm{C}), 139.5(\mathrm{CH}), 134.2(\mathrm{CH})$, $132.3(\mathrm{C}), 129.1(\mathrm{CH}), 129.0(\mathrm{CH}), 61.4(\mathrm{CH}), 48.6(\mathrm{CH}), 47.5\left(\mathrm{CH}_{2}\right), 45.5(\mathrm{CH}), 45.4(\mathrm{CH}) . \mathrm{MS}$ (CI) $233\left(\mathrm{MH}^{+}\right)$; $\mathrm{HRMS}\left(\mathrm{FAB}^{+}\right)$calcd. for $\mathrm{C}_{14} \mathrm{H}_{14} \mathrm{ClO}\left(\mathrm{MH}^{+}\right)$233.0733; found 233.0816.

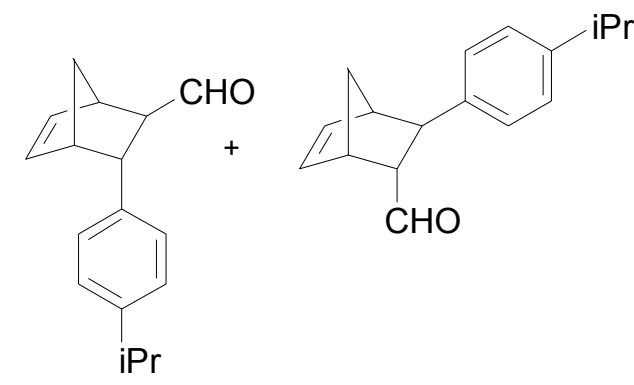




\section{$(1 R, 2 R, 3 R, 4 S)$-3-(4-isopropylphenyl)-bicyclo[2.2.1]hept-5-ene-2-carboxaldehyde} and $(1 S, 2 S, 3 S, 4 R)$-3-(4-isopropylphenyl)-bicyclo[2.2.1]hept-5-ene-2-carboxaldehyde (Table 3, entry, 4). Prepared according to the general procedure described above from $(E)-4$ isopropylcinnamaldehyde (75 $\mathrm{mg}, 0.43 \mathrm{mmol})$ and cyclopentadiene (85 $\mathrm{mg}, 1.3 \mathrm{mmol})$. Purification by silica gel chromatography (5\% EtOAc in hexanes) provided the desired material as a 1.7:1 mixture of endo and exo isomers (colourless oil, $87 \mathrm{mg}, 84 \%$ ). Endo ee 90\%. Enantiomeric ratios were determined by acetalization with $(+)-(R, R)$-hydrobenzoin and ${ }^{1} \mathrm{H} \mathrm{NMR}$ analysis: $\left(500 \mathrm{MHz}, \mathrm{C}_{6} \mathrm{D}_{6}\right)$ exo isomers chemical shift were identical, endo isomers $\delta 5.35$ (d, $\mathrm{J}=8.1 \mathrm{~Hz}, \mathrm{CHO}_{2}$, major isomer), $5.31\left(\mathrm{~d}, \mathrm{~J}=8.2 \mathrm{~Hz}, \mathrm{CHO}_{2}\right.$, minor isomer). $[\alpha]_{\mathrm{D}}-170.6$ (c 1.09 $\mathrm{CHCl}_{3}$ ); IR (neat) $1713 \mathrm{~cm}^{-1}$; exo isomer ${ }^{1} \mathrm{H} \mathrm{NMR}\left(500 \mathrm{MHz}, \mathrm{CDCl}_{3}\right) \delta 9.91(\mathrm{~d}, \mathrm{~J}=2.0 \mathrm{~Hz}, 1 \mathrm{H})$, $7.11(\mathrm{~d}, \mathrm{~J}=8.0 \mathrm{~Hz}, 2 \mathrm{H}), 7.07(\mathrm{~d}, \mathrm{~J}=8.0 \mathrm{~Hz}, 2 \mathrm{H}), 6.32(\mathrm{dd}, \mathrm{J}=5.5,3.0 \mathrm{~Hz}, 1 \mathrm{H}), 6.08(\mathrm{dd}, \mathrm{J}=$ 5.5, $2.5 \mathrm{~Hz}, 1 \mathrm{H}), 3.67$ (dd, J = 5.5, 4.0 Hz, 1H), 3.20 (br, 2H), 2.90-2.83 (m, 1H), 2.58-2.56 (m, $1 \mathrm{H}), 1.61-1.59(\mathrm{~m}, 1 \mathrm{H}), 1.55-1.53(\mathrm{~m}, 1 \mathrm{H}), 1.21(\mathrm{~d}, \mathrm{~J}=7.0 \mathrm{~Hz}, 6 \mathrm{H}) ;{ }^{13} \mathrm{C} \mathrm{NMR}(125 \mathrm{MHz}$, $\left.\mathrm{CDCl}_{3}\right) \delta 202.9(\mathrm{CH}), 146.8(\mathrm{C}), 139.8(\mathrm{C}), 139.1(\mathrm{CH}), 136.5(\mathrm{CH}), 127.7(\mathrm{CH}), 126.1(\mathrm{CH})$, $59.5(\mathrm{CH}), 48.4(\mathrm{CH}), 47.5(\mathrm{CH}), 45.4(\mathrm{CH}), 45.0(\mathrm{CH}), 33.5(\mathrm{CH}), 23.9\left(\mathrm{CH}_{3}\right)$; endo isomer ${ }^{1} \mathrm{H}$ $\operatorname{NMR}\left(500 \mathrm{MHz}, \mathrm{CDCl}_{3}\right) \delta 9.58(\mathrm{~d}, \mathrm{~J}=2.0 \mathrm{~Hz}, 1 \mathrm{H}), 7.19(\mathrm{~d}, \mathrm{~J}=8.5 \mathrm{~Hz}, 2 \mathrm{H}), 7.16(\mathrm{~d}, \mathrm{~J}=8.5 \mathrm{~Hz}$, 2H), $6.40(\mathrm{dd}, \mathrm{J}=5.5,2.5 \mathrm{~Hz}, 1 \mathrm{H}), 6.15(\mathrm{dd}, \mathrm{J}=5.5,2.5 \mathrm{~Hz}, 1 \mathrm{H}), 3.31$ (br, 1H), 3.09 (br, 1H), 3.05-3.04 (m, 1H), 2.98-2.96 (m, 1H), 2.90-2.83 (m, 1H), 1.80 (d, J = 8.5 Hz, 1H), 1.61-1.60 (m, 1H), $1.23(\mathrm{~d}, \mathrm{~J}=8.0 \mathrm{~Hz}, 6 \mathrm{H}) ;{ }^{13} \mathrm{C} \mathrm{NMR}\left(125 \mathrm{MHz}, \mathrm{CDCl}_{3}\right) \delta 203.6(\mathrm{CH}), 146.7$ (C), 140.7 (C), $136.1(\mathrm{CH}), 133.6(\mathrm{CH}), 127.2(\mathrm{CH}), 126.5(\mathrm{CH}), 60.6(\mathrm{CH}), 48.5(\mathrm{CH}), 47.0\left(\mathrm{CH}_{2}\right), 45.3(\mathrm{CH})$, $45.0(\mathrm{CH}), 33.5,(\mathrm{CH}), 23.9\left(\mathrm{CH}_{3}\right) . \mathrm{MS}\left(\mathrm{FAB}^{+}\right)\left(\mathrm{MH}^{+}\right)$241.2.

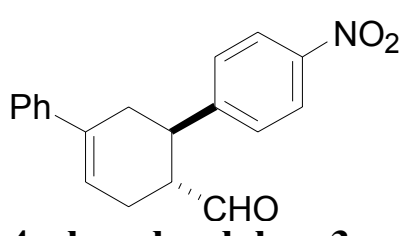

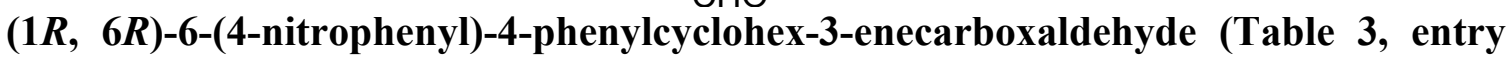
5). Prepared according to the general procedure described above from (E)-4nitrocinnamaldehyde $(50 \mathrm{mg}, 0.28 \mathrm{mmol})$ and 1-(methylene-allyl)-benzene ${ }^{5}$ (146 $\mathrm{mg}, 1.12$ mmol). Purification by silica gel chromatography (15\% EtOAc in hexanes) provided the material as a pale yellow solid (74 mg, $86 \%$ ). Mp 159-161 ${ }^{\circ} \mathrm{C} ; 85 \%$ ee. Enantiomeric ratio was

\footnotetext{
${ }^{5}$ Hawkins, E. G. E.; Thompson, R. D. J. Chem. Soc. 1961, 370.
} 
determined by acetalization with $(+)-(R, R)$-hydrobenzoin and ${ }^{1} \mathrm{H}$ NMR analysis: $(500 \mathrm{MHz}$, $\left.\mathrm{C}_{6} \mathrm{D}_{6}\right) \delta 5.29\left(\mathrm{~d}, \mathrm{~J}=3.1 \mathrm{~Hz}, \mathrm{CHO}_{2}\right.$, major isomer), $5.13\left(\mathrm{~d}, \mathrm{~J}=2.4 \mathrm{~Hz}, \mathrm{CHO}_{2}\right.$, minor isomer). $[\alpha]_{\mathrm{D}}$ $+8.6\left(c 0.88 \mathrm{CHCl}_{3}\right) ; \mathrm{IR}$ (neat) 1724, 1597, 1519, $1350 \mathrm{~cm}^{-1} ;{ }^{1} \mathrm{H} \mathrm{NMR}\left(500 \mathrm{MHz}, \mathrm{CDCl}_{3}\right) \delta 9.57$ (d, J = 2.2 Hz, 1H), 8.18 (d, J = 8.5 Hz, 2H), 7.43 (d, J = 9.3 Hz, 2H), 7.37-7.25 (m, 5H), 6.21 (br, 1H), 3.45 (ddd, J = 9.0, 9.0, $5.6 \mathrm{~Hz}, 1 \mathrm{H}), 2.96-2.91(\mathrm{~m}, 1 \mathrm{H}), 2.84-2.80(\mathrm{~m}, 1 \mathrm{H}), 2.66-2.43$ (m, 3H); ${ }^{13} \mathrm{C}$ NMR (125 MHz, $\left.\mathrm{CDCl}_{3}\right) \delta 202.6(\mathrm{CH}), 151.0(\mathrm{C}), 146.8(\mathrm{C}), 140.4(\mathrm{C}), 136.1(\mathrm{C})$, $128.7(\mathrm{CH}), 128.4(\mathrm{CH}), 127.4(\mathrm{CH}), 124.9(\mathrm{CH}), 124.0(\mathrm{CH}), 121.5(\mathrm{CH}), 50.6(\mathrm{CH}), 40.3$ $(\mathrm{CH}), 33.9(\mathrm{CH}), 25.0(\mathrm{CH})$; MS (EI) 307.1 $\left(\mathrm{M}^{+}\right)$; HRMS calcd. for $\mathrm{C}_{19} \mathrm{H}_{17} \mathrm{NO}_{3}\left(\mathrm{M}^{+}\right)$307.1209; found 307.1191 .

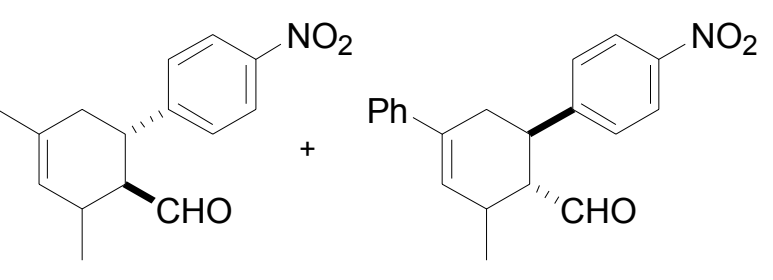

$(1 R, 6 R)$-2,4-Dimethyl-6-(4-nitrophenyl)-cyclohex-3-enecarboxaldehyde and (1S, 6S)-2,4-Dimethyl-6-(4-nitrophenyl)-cyclohex-3-enecarboxaldehyde (Table 3, entry 6). Prepared according to the general procedure described above from $(E)$-4-nitrocinnamaldehyde (100 mg $0.564 \mathrm{mmol}$ ) and trans-2-methyl-1,3-pentadiene (231 mg, $2.82 \mathrm{mmol})$ using $\mathrm{CH}_{2} \mathrm{Cl}_{2}$ in place of ether during the extraction. Purification by silica gel chromatography (5\% EtOAc in hexanes) provided the desired material as a 1.9:1 mixture of exo and endo isomers (pale yellow solid, $102 \mathrm{mg}, 71 \%$ ). Mp $92-97{ }^{\circ} \mathrm{C}$; Exo ee $24 \%$ endo ee $69 \%$. Enantiomeric ratios were determined using chiral GLC analysis (Agilent $/ \mathrm{J} \& \mathrm{~W}$ CycloSil-B, $210^{\circ} \mathrm{C}$ isotherm, flow $=3.0$ $\mathrm{mL} / \mathrm{min}$ ) exo isomers $\mathrm{t}_{\mathrm{r}}=24.7 \mathrm{~min}, 28.6 \mathrm{~min}$, endo isomers $\mathrm{t}_{\mathrm{r}}=23.8 \mathrm{~min}, 27.3 \mathrm{~min} ;[\alpha]_{\mathrm{D}}-38.7$ (c $\left.0.85 \mathrm{CHCl}_{3}\right)$; IR (neat) $1720,1595,1515,1340 \mathrm{~cm}^{-1}$; exo isomer ${ }^{1} \mathrm{H} \mathrm{NMR}\left(500 \mathrm{MHz}, \mathrm{CDCl}_{3}\right) \delta$ $9.63(\mathrm{~d}, \mathrm{~J}=2.9 \mathrm{~Hz}, 1 \mathrm{H}), 8.16-8.13(\mathrm{~m}, 2 \mathrm{H}), 7.36-7.33(\mathrm{~m}, 2 \mathrm{H}), 5.48-5.47$ (br, $1 \mathrm{H}), 3.44-3.40$ (m, 1H), $2.84(\mathrm{ddd}, \mathrm{J}=8.3,5.4,2.9 \mathrm{~Hz}, 1 \mathrm{H}), 2.61(\mathrm{br}, 1 \mathrm{H}), 2.29(\mathrm{dd}, \mathrm{J}=18.1,6.3 \mathrm{~Hz}, 1 \mathrm{H}), 2.20-2.01$ (m, 1H), $1.70(\mathrm{~s}, 3 \mathrm{H}), 1.04(\mathrm{~d}, \mathrm{~J}=6.9 \mathrm{~Hz}, 3 \mathrm{H}) ;\left(125 \mathrm{MHz}, \mathrm{CDCl}_{3}\right) \delta 204.0(\mathrm{CH}), 152.1(\mathrm{C})$, $146.5(\mathrm{C}), 132.16(\mathrm{C}), 128.2(\mathrm{CH}), 126.1(\mathrm{CH}), 123.7(\mathrm{CH}), 58.8(\mathrm{CH}), 37.0(\mathrm{CH}), 36.6\left(\mathrm{CH}_{2}\right)$, $29.5(\mathrm{CH}), 23.0\left(\mathrm{CH}_{3}\right), 17.1\left(\mathrm{CH}_{3}\right)$, endo isomer ${ }^{1} \mathrm{H} \mathrm{NMR}\left(125 \mathrm{MHz}, \mathrm{CDCl}_{3}\right) \delta 9.39(\mathrm{~d}, \mathrm{~J}=4.9$ $\mathrm{Hz}, 1 \mathrm{H}), 8.16-8.13(\mathrm{~m}, 2 \mathrm{H}), 7.36-7.33(\mathrm{~m}, 2 \mathrm{H}), 5.31$ (br, 1H), 3.22 (ddd, J = 11.2, 11.2, 5.9 Hz, 1H), $2.61(\mathrm{br}, 1 \mathrm{H}), 2.43(\mathrm{ddd}, \mathrm{J}=11.4,10.2,4.4 \mathrm{~Hz}, 1 \mathrm{H}), 2.20-2.01(\mathrm{~m}, 2 \mathrm{H}), 1.71(\mathrm{~s}, 3 \mathrm{H}), 1.02$ $(\mathrm{d}, \mathrm{J}=7.3 \mathrm{~Hz}, 3 \mathrm{H}) ;\left(125 \mathrm{MHz}, \mathrm{CDCl}_{3}\right) \delta 203.7(\mathrm{CH}), 150.1(\mathrm{C}), 146.5(\mathrm{C}), 132.1(\mathrm{C}), 128.3$ 
$(\mathrm{CH}), 125.6(\mathrm{CH}), 124.0(\mathrm{CH}), 54.8(\mathrm{CH}), 41.9(\mathrm{CH}), 38.2\left(\mathrm{CH}_{2}\right), 31.5(\mathrm{CH}), 22.9\left(\mathrm{CH}_{3}\right), 19.9$ $\left(\mathrm{CH}_{3}\right)$, MS $259.1\left(\mathrm{M}^{+}\right)$; HRMS calcd. for $\mathrm{C}_{15} \mathrm{H}_{17} \mathrm{NO}_{3}\left(\mathrm{M}^{+}\right)$259.1208; found 259.1196.

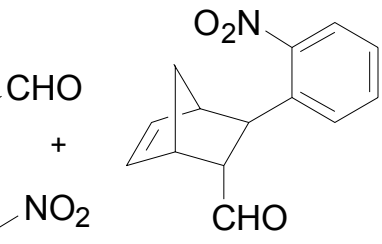

$(1 R, 2 R, 3 R, 4 S)$-3-(2-Nitrophenyl)-bicyclo[2.2.1] hept-5-ene-2-carboxaldehyde and $(1 S, 2 S, 3 S, 4 R)$-3-(2-Nitrophenyl)-bicyclo[2.2.1] hept-5-ene-2-carboxaldehyde (Table 3, entry 7). Prepared according to the general procedure described above from (E)-2nitrocinnamaldehyde (100 mg $0.564 \mathrm{mmol})$ and cyclopentadiene (112 mg, $1.69 \mathrm{mmol})$. Purification by silica gel chromatography (15\% EtOAc in hexanes) provided the desired material as a 1.2:1 mixture of exo and endo isomers (pale yellow oil, $123 \mathrm{mg}, 90 \%$ ). Exo ee $87 \%$, endo ee $86 \%$. Enantiomeric ratios were determined by acetalization with $(+)-(R, R)$-hydrobenzoin and ${ }^{1} \mathrm{H}$ NMR analysis: (500 MHz, $\mathrm{C}_{6} \mathrm{D}_{6}$ ) exo isomers $\delta 5.55$ (d, J=5.2 Hz, $\mathrm{CHO}_{2}$, major isomer), 5.51 (d, $\mathrm{J}=6.3 \mathrm{~Hz}, \mathrm{CHO}_{2}$, minor isomer), endo isomers $\delta 5.22$ (d, J=8.1 Hz, $\mathrm{CHO}_{2}$, major isomer), 5.14 (d, J=8.3 Hz, $\mathrm{CHO}_{2}$, minor isomer). $[\alpha]_{\mathrm{D}}+1.86\left(c 1.40 \mathrm{CHCl}_{3}\right.$ ); IR (Neat) $1713 \mathrm{~cm}^{-1} ;{ }^{13} \mathrm{C} \mathrm{NMR}$ (125 MHz, $\left.\mathrm{CDCl}_{3}\right) \delta 203.5(\mathrm{CH}), 201.4(\mathrm{CH}), 151.0(\mathrm{C}), 150.2(\mathrm{C}), 139.1(\mathrm{CH}), 137.2(\mathrm{C})$, $136.8(\mathrm{CH}), 136.2(\mathrm{CH}), 135.9(\mathrm{C}), 134.0(\mathrm{CH}), 132.8(\mathrm{CH}), 131.6(\mathrm{CH}), 128.6(\mathrm{CH}), 127.9$ $(\mathrm{CH}), 127.3(\mathrm{CH}), 127.1(\mathrm{CH}), 124.7(\mathrm{CH}), 123.8(\mathrm{CH}), 59.1(\mathrm{CH}), 58.9(\mathrm{CH}), 49.7(\mathrm{CH}), 49.1$ $(\mathrm{CH}), 48.0\left(\mathrm{CH}_{2}\right), 47.1(\mathrm{CH}), 46.5(\mathrm{CH}), 46.1(\mathrm{CH}), 41.5(\mathrm{CH}), 39.9(\mathrm{CH})$; exo isomer ${ }^{1} \mathrm{H}$ NMR $\left(500 \mathrm{MHz}, \mathrm{CDCl}_{3}\right) \delta 9.78(\mathrm{~d}, \mathrm{~J}=2.3 \mathrm{~Hz}, 1 \mathrm{H}), 7.69(\mathrm{~d}, \mathrm{~J}=8.3 \mathrm{~Hz}, 1 \mathrm{H}), 7.57-7.50(\mathrm{~m}, 1 \mathrm{H}), 7.43-$ $7.24(\mathrm{~m}, 1 \mathrm{H}), 7.16-7.14(\mathrm{~d}, \mathrm{~J}=7.2 \mathrm{~Hz}, 1 \mathrm{H}), 6.48-6.44(\mathrm{~m}, 1 \mathrm{H}), 5.99$ (dd, J = 5.4, $2.4 \mathrm{~Hz}, 1 \mathrm{H})$, 4.07-4.06 (m, 1H), $3.54(\mathrm{~s}, 1 \mathrm{H}), 3.26(\mathrm{~s}, 1 \mathrm{H}), 2.59-2.58(\mathrm{~m}, 1 \mathrm{H}), 1.65-1.63(\mathrm{~m}, 1 \mathrm{H}), 1.55(\mathrm{~d}, \mathrm{~J}=$ $9.2 \mathrm{~Hz}, 1 \mathrm{H})$; endo isomer ${ }^{1} \mathrm{H}$ NMR $\left(500 \mathrm{MHz}, \mathrm{CDCl}_{3}\right) \delta 9.37(\mathrm{~d}, \mathrm{~J}=3.4 \mathrm{~Hz}, 1 \mathrm{H}), 7.79(\mathrm{~d}, \mathrm{~J}=$ 7.8 Hz, 1H), 7.57-7.50 (m, 1H), 7.43-7.24 (m, 2H), 6.48-6.44 (m, 1H), 6.19 (dd, J = 5.4, $2.9 \mathrm{~Hz}$, 1H), $3.41(\mathrm{~d}, \mathrm{~J}=4.9 \mathrm{~Hz}, 1 \mathrm{H}), 3.30(\mathrm{~s}, 1 \mathrm{H}), 3.10(\mathrm{~s}, 1 \mathrm{H}), 2.93-2.91(\mathrm{~m}, 1 \mathrm{H}), 1.81(\mathrm{~d}, \mathrm{~J}=9.1 \mathrm{~Hz}$, $1 \mathrm{H}), 1.63(\mathrm{~d}, \mathrm{~J}=8.9 \mathrm{~Hz}, 1 \mathrm{H})$. MS (ESI) $\left(\mathrm{MH}^{+}\right) 244.2$. 


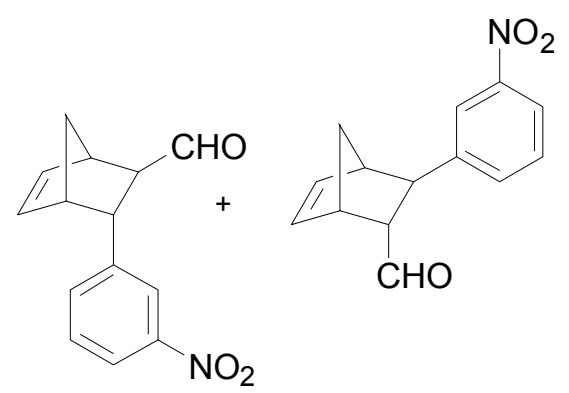

$(1 R, 2 R, 3 R, 4 S)$-3-(3-Nitrophenyl)-bicyclo[2.2.1] hept-5-ene-2-carboxaldehyde and (1S, 2S, 3S, 4R)-3-(3-Nitrophenyl)-bicyclo[2.2.1] hept-5-ene-2-carboxaldehyde (Table 3, entry 8). Prepared according to the general procedure described above from (E)-3nitrocinnamaldehyde (100 mg, $0.564 \mathrm{mmol})$ and cyclopentadiene (112 mg, $1.69 \mathrm{mmol})$ using $\mathrm{CH}_{2} \mathrm{Cl}_{2}$ in place of ether during the extraction. Purification by silica gel chromatography (15\% EtOAc in hexanes) provided the desired material as a 2.1:1 mixture of exo and endo isomers (colourless oil, $121 \mathrm{mg}, 88 \%$ ). Exo ee 90\%, endo ee 94\%. Enantiomeric ratios were determined by acetalization with $(+)-(R, R)$-hydrobenzoin and ${ }^{1} \mathrm{H}$ NMR analysis: $\left(500 \mathrm{MHz}, \mathrm{C}_{6} \mathrm{D}_{6}\right)$ exo isomers $\delta 5.46\left(\mathrm{~d}, \mathrm{~J}=4.5 \mathrm{~Hz}, \mathrm{CHO}_{2}\right.$, major isomer), $5.38\left(\mathrm{~d}, \mathrm{~J}=5.1 \mathrm{~Hz}, \mathrm{CHO}_{2}\right.$, minor isomer) endo isomers $\delta 5.09$ (d, J=7.6 Hz, $\mathrm{CHO}_{2}$, major isomer), $5.00\left(\mathrm{~d}, \mathrm{~J}=7.6 \mathrm{~Hz}, \mathrm{CHO}_{2}\right.$, minor isomer). $[\alpha]_{\mathrm{D}}-149\left(\right.$ c $\left.1.24 \mathrm{CHCl}_{3}\right)$; IR (neat) $1713 \mathrm{~cm}^{-1}$; exo isomer ${ }^{1} \mathrm{H} \mathrm{NMR}\left(300 \mathrm{MHz}, \mathrm{CDCl}_{3}\right) \delta 9.90$ $(\mathrm{d}, \mathrm{J}=1.5 \mathrm{~Hz}, 1 \mathrm{H}), 8.11-7.95(\mathrm{~m}, 2 \mathrm{H}), 7.59-7.37(\mathrm{~m}, 2 \mathrm{H}), 6.43-6.40(\mathrm{~m}, 1 \mathrm{H}), 6.03$ (dd, J = 5.7, $3.0 \mathrm{~Hz}, 1 \mathrm{H}), 3.86(\mathrm{dd}, \mathrm{J}=4.5,3.9 \mathrm{~Hz}, 1 \mathrm{H}), 3.28-3.15(\mathrm{~m}, 2 \mathrm{H}), 2.62(\mathrm{dd}, \mathrm{J}=5.1,0.6 \mathrm{~Hz}, 1 \mathrm{H})$, 1.70-1.66 (m, 1H), 1.61-1.57 (m, 1H); ${ }^{13} \mathrm{C}$ NMR $\left(75 \mathrm{MHz}, \mathrm{CDCl}_{3}\right) \delta 201.5(\mathrm{CH}), 148.1(\mathrm{C})$, 144.8 (C), 137.1 (CH), $135.9(\mathrm{CH}), 134.3(\mathrm{CH}), 129.0(\mathrm{CH}), 122.5(\mathrm{CH}), 121.5(\mathrm{CH}), 59.6(\mathrm{CH})$, $48.4(\mathrm{CH}), 47.5\left(\mathrm{CH}_{2}\right), 45.5(\mathrm{CH}), 44.7(\mathrm{CH})$; endo isomer ${ }^{1} \mathrm{H}$ NMR $\left(300 \mathrm{MHz}, \mathrm{CDCl}_{3}\right) \delta 9.62$ $(\mathrm{d}, \mathrm{J}=1.8 \mathrm{~Hz}, 1 \mathrm{H}), 8.11-7.95(\mathrm{~m}, 2 \mathrm{H}), 7.59-7.37(\mathrm{~m}, 2 \mathrm{H}), 6.43-6.40(\mathrm{~m}, 1 \mathrm{H}), 6.17(\mathrm{dd}, \mathrm{J}=5.7$, $2.7 \mathrm{~Hz}, 1 \mathrm{H}), 3.41$ (br, 1H), 3.28-3.15 (m, 2H), 2.98-2.95 (m, 1H), 1.78-1.75 (m, 1H), 1.61-1.57 (m, 1H); ${ }^{13} \mathrm{C}$ NMR (75 MHz, $\left.\mathrm{CDCl}_{3}\right) \delta 202.2(\mathrm{CH}), 148.1(\mathrm{C}), 145.9(\mathrm{C}), 139.1(\mathrm{CH}), 134.3$ $(\mathrm{CH}), 133.9(\mathrm{CH}), 129.5(\mathrm{CH}), 122.5(\mathrm{CH}), 121.4(\mathrm{CH}), 61.0(\mathrm{CH}), 48.2(\mathrm{CH}), 47.1\left(\mathrm{CH}_{2}\right), 45.2$ $(\mathrm{CH}), 45.0(\mathrm{CH})$.

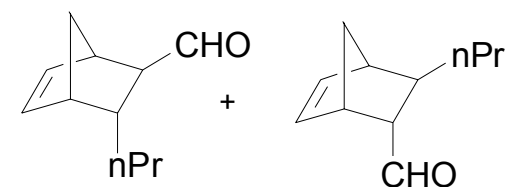

$(1 R, 2 R, 3 R, 4 S)$-3-propyl-bicyclo[2.2.1] hept-5-ene-2-carboxaldehyde and $(1 S, 2 S$, 3S, 4R)-3-propyl-bicyclo[2.2.1]hept-5-ene-2-carboxaldehyde (Table 3, entry 9). Prepared 
according to the general procedure described above from $(E)$-2-hexenal $(50 \mathrm{mg}, 0.51 \mathrm{mmol})$ and cyclopentadiene $(101 \mathrm{mg}, 1.53 \mathrm{mmol})$. Purification by silica gel chromatography $(5 \%$ EtOAc in hexanes) provided a colourless oil (69 mg, $83 \%)$; 1.6:1 exo:endo, exo ee $81 \%$ endo ee $75 \%$. Enantiomeric ratios were determined using chiral GLC analysis (Agilent/J\&W CycloSil-B, 100 ${ }^{\circ} \mathrm{C}$ hold $3 \mathrm{~min}$ then $1{ }^{\circ} \mathrm{C} / \mathrm{min}$ gradient, flow $=3.0 \mathrm{~mL} / \mathrm{min}$ ) exo isomers $\mathrm{t}_{\mathrm{r}}=26.2 \mathrm{~min}, 27.6 \mathrm{~min}$, endo isomers $\mathrm{t}_{\mathrm{r}}=26.9 \mathrm{~min}, 28.8 \mathrm{~min} .{ }^{1} \mathrm{H} \mathrm{NMR},{ }^{13} \mathrm{C} \mathrm{NMR}$ and IR data were identical to those previously reported. ${ }^{6}$

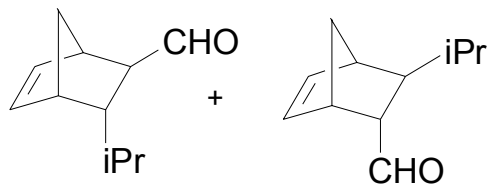

$((1 R, 2 R, 3 R, 4 S)$-3-isopropyl-bicyclo[2.2.1]hept-5-ene-2-carboxaldehyde and $(1 S, 2 S$, 3S, 4R)-3-isopropyl-bicyclo[2.2.1] hept-5-ene-2-carboxaldehyde (Table 3, entry 10). Prepared according to the general procedure described above from $(E)$-4-methyl-2-pentenal $(85 \mathrm{mg}, 0.87$ $\mathrm{mmol})$ and cyclopentadiene $(171 \mathrm{mg}, 2.60 \mathrm{mmol})$. Purification by silica gel chromatography $(5 \%$ EtOAc in hexanes) provided a colourless oil (120 mg, $84 \%$ ). 2.6:1 exo:endo, exo ee $85 \%$, endo ee $84 \%$. Enantiomeric ratios were determined by reduction (4 eq. of $\mathrm{NaBH}_{4}$ in $\mathrm{MeOH}$ ) and subjection of to the corresponding alcohols to chiral GLC analysis (Agilent/J\&W CycloSil-B, $100{ }^{\circ} \mathrm{C}$ hold $3 \mathrm{~min}$ then $1{ }^{\circ} \mathrm{C} / \mathrm{min}$ gradient, flow $=3.0 \mathrm{~mL} / \mathrm{min}$ ) exo isomers $\mathrm{t}_{\mathrm{r}}=39.6 \mathrm{~min}, 42.2 \mathrm{~min}$, endo isomers $\mathrm{t}_{\mathrm{r}}=38.9 \mathrm{~min}, 39.3 \mathrm{~min} .{ }^{1} \mathrm{H} \mathrm{NMR},{ }^{13} \mathrm{C} \mathrm{NMR}$ and IR data were identical to those previously reported. ${ }^{6}$

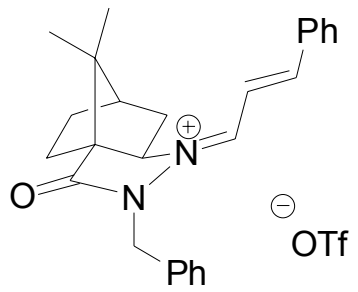

NMR study of the inimium ion formed from $(S)-(+)-3-B e n z y l-10,10-d i m e t h y l-3,4-$ diaza-tricyclo $\left[5.2 .1 .0^{1,5}\right]$ decan-2-one $(5),(E)$-cinnamaldehyde and triflic acid. To a solution of (E)-cinnamaldehyde (12.2 mg, $0.092 \mathrm{mmol})$ and $(S)-(+)-3$-Benzyl-10,10-dimethyl-3,4-diazatricyclo [5.2.1.0 ${ }^{1,5}$ ] decan-2-one $(25.0 \mathrm{mg}, 0.092 \mathrm{mmol})$ in $\mathrm{CD}_{3} \mathrm{NO}_{2}(1.0 \mathrm{~mL})$ was added $\mathrm{CF}_{3} \mathrm{SO}_{3} \mathrm{H}(8.2 \mu \mathrm{L}, 0.09 \mathrm{mmol})$. The reaction was monitored at room temperature by ${ }^{1} \mathrm{H}$ NMR using 2, 5 and 10 minute intervals until equilibrium was reached. ${ }^{1} \mathrm{H}$ NMR $\left(300 \mathrm{MHz}, \mathrm{CD}_{3} \mathrm{NO}_{2}\right)$

\footnotetext{
${ }^{6}$ Ahrendt, K. A.; Borths, C. J.; MacMillan, D.W.C. J. Am. Chem. Soc. 2000, 122, 4243.
} 
$\delta 8.22(\mathrm{dd}, \mathrm{J}=10.2,2.1 \mathrm{~Hz}, 1 \mathrm{H}), 7.81(\mathrm{~d}, \mathrm{~J}=15.3 \mathrm{~Hz}, 1 \mathrm{H}), 7.77-7.40(\mathrm{~m}, 2 \mathrm{H}), 7.59-7.42(\mathrm{~m}$, 8H), 7.12 (dd, J=15.3, 10.5 Hz, 1H), 5.31 (d, J=17.0 Hz, 1H), 5.27 (d, J=17.0 Hz, 1H), 5.04-4.99 (m, 1H), 2.84-2.73 (m, 1H), $2.66(\mathrm{dd}, \mathrm{J}=13.5,8.4 \mathrm{~Hz}, 1 \mathrm{H}), 2.42$ (ddd, J = 11.9, 11.9, $5.4 \mathrm{~Hz}$, 1H), 2.23-2.19 (m, 2H), 1.84-1.76 (m, 1H), 1.66-1.57 (m, 1H), 1.22 (s, 3H), 1.06 (s, 3H).

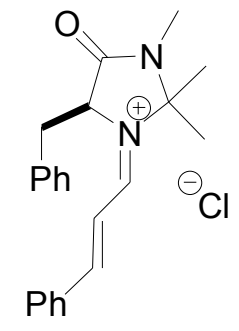

NMR study of the inimium ion formed from (5S)-2,2,3-trimethyl-5-phenylmethyl-4imidazolidinone monohydrochloride (10) and (E)-cinnamaldehyde. To a solution of (E)cinnamaldehyde (13.0 mg, $0.098 \mathrm{mmol})$ in $\mathrm{CD}_{3} \mathrm{OD}(1.0 \mathrm{~mL})$ was added (5S)-2,2,3-trimethyl-5phenylmethyl-4-imidazolidinone monohydrochloride $(25.0 \mathrm{mg}, 0.098 \mathrm{mmol})$. The reaction was monitored at room temperature by ${ }^{1} \mathrm{H}$ NMR using 2, 5 and 10 minute intervals until equilibrium was reached. Only peaks assigned to the iminium salt are reported. ${ }^{1} \mathrm{H}$ NMR $\left(300 \mathrm{MHz}, \mathrm{CD}_{3} \mathrm{OD}\right)$ $\delta 9.39$ (d, J=10.6 Hz, 1H), 8.39 (d, J=14.9 Hz,1H), 8.07-8.02 (d, 1H), 7.76-7.2 (m, 7H), 7.137.07 (m, 2H), 5.57-5.50 (br, 1H), 3.67 (dd, J=14.8, 5.6, 1H), 3.58-3.53 (m, 1H), $2.89(\mathrm{~s}, 3 \mathrm{H})$, $1.82(\mathrm{~s}, 3 \mathrm{H}), 0.89(\mathrm{~s}, 3 \mathrm{H})$. 


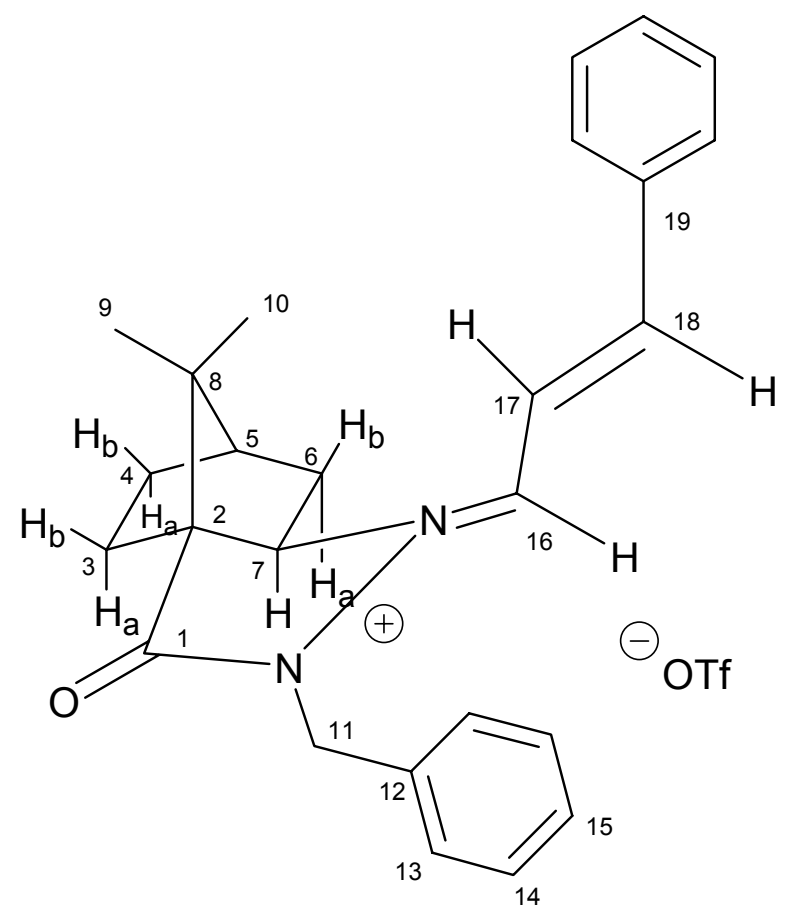

\begin{tabular}{clll}
\hline No & \multicolumn{1}{c}{$1_{\mathrm{H} \text { shift }, \delta(\mathrm{m}, \mathrm{J} \mathrm{Hz})}$} & \multicolumn{1}{c}{ COSY } & \multicolumn{1}{c}{ NOESY } \\
\hline $3 \mathrm{a}$ & $1.84-1.76(\mathrm{~m}, 1 \mathrm{H})$ & $3 \mathrm{~b}, 4 \mathrm{a}$ & $3 \mathrm{~b}, 4 \mathrm{a}(\mathrm{w}), 7$ \\
$3 \mathrm{~b}$ & $2.42(\mathrm{ddd}, \mathrm{J}=11.9,11.9,5.4 \mathrm{~Hz}, 1 \mathrm{H})$ & $3 \mathrm{a}, 4 \mathrm{a}, 4 \mathrm{~b}$ & $3 \mathrm{a}, 4 \mathrm{~b}(\mathrm{w})$ \\
$4 \mathrm{a}$ & $1.66-1.57(\mathrm{~m}, 1 \mathrm{H})$ & $4 \mathrm{~b}, 5$ & $3 \mathrm{a}(\mathrm{w}), 4 \mathrm{~b}, 5,7$ \\
$4 \mathrm{~b}$ & $2.23-2.19(\mathrm{~m}, 1 \mathrm{H})$ & $4 \mathrm{a}, 3 \mathrm{~b}$ & $3 \mathrm{a}(\mathrm{w}), 4 \mathrm{a}, 5,9$ \\
5 & $2.23-2.19(\mathrm{~m}, 1 \mathrm{H})$ & $4 \mathrm{~b}, 6 \mathrm{~b}$ & $4 \mathrm{a}, 4 \mathrm{~b}, 6 \mathrm{a}, 6 \mathrm{~b}, 9,10(\mathrm{w})$ \\
$6 \mathrm{a}$ & $2.66(\mathrm{dd}, \mathrm{J}=13.5,8.4 \mathrm{~Hz}, 1 \mathrm{H})$ & $6 \mathrm{~b}, 7$ & $5,6 \mathrm{~b}, 7,17$ \\
$6 \mathrm{~b}$ & $2.84-2.73(\mathrm{~m}, 1 \mathrm{H})$ & $6 \mathrm{a}, 5$ & $5,6 \mathrm{a}, 17$ \\
7 & $5.04-4.99(\mathrm{~m}, 1 \mathrm{H})$ & $6 \mathrm{a}$ & $3 \mathrm{a}, 4 \mathrm{a}, 6 \mathrm{a}, 17$ \\
9 & $1.22(\mathrm{~s}, 3 \mathrm{H})$ & & $3 \mathrm{~b}, 4 \mathrm{~b}, 5,10$ \\
10 & $1.06(\mathrm{~s}, 3 \mathrm{H})$ & & $5,6 \mathrm{~b}, 16(\mathrm{w})$ \\
$11 \mathrm{a}$ & $5.31(\mathrm{~d}, \mathrm{~J}=17.0 \mathrm{~Hz}, 1 \mathrm{H})$ & $11 \mathrm{~b}$ & $11 \mathrm{~b}, 16$ \\
$11 \mathrm{~b}$ & $5.27(\mathrm{~d}, \mathrm{~J}=17.0 \mathrm{~Hz}, 1 \mathrm{H})$ & $11 \mathrm{a}$ & $11 \mathrm{a}, 16$ \\
16 & $8.22(\mathrm{dd}, \mathrm{J}=10.2,2.1 \mathrm{~Hz}, 1 \mathrm{H})$ & $7(\mathrm{w}), 17$ & $7(\mathrm{w}), 11,10(\mathrm{w}), 17(\mathrm{w})$, \\
& & & 18 \\
17 & $7.12(\mathrm{dd}, \mathrm{J}=15.3,10.5 \mathrm{~Hz}, 1 \mathrm{H})$ & 16,18 & $6 \mathrm{a}, 6 \mathrm{~b}, 7,16(\mathrm{w})$ \\
18 & $7.81(\mathrm{~d}, \mathrm{~J}=15.3 \mathrm{~Hz}, 1 \mathrm{H})$ & 17 & 16 \\
\hline
\end{tabular}


$\mathrm{O} \mathrm{OH} \quad \mathrm{H}$ 
$\sum_{\mathrm{OH}}^{\mathrm{N}}{ }_{\mathrm{H}}^{\mathrm{N}}$ 
$\overbrace{\substack{\mathrm{N} \\ \mathrm{CH}_{3}}}^{\mathrm{N}}$ 

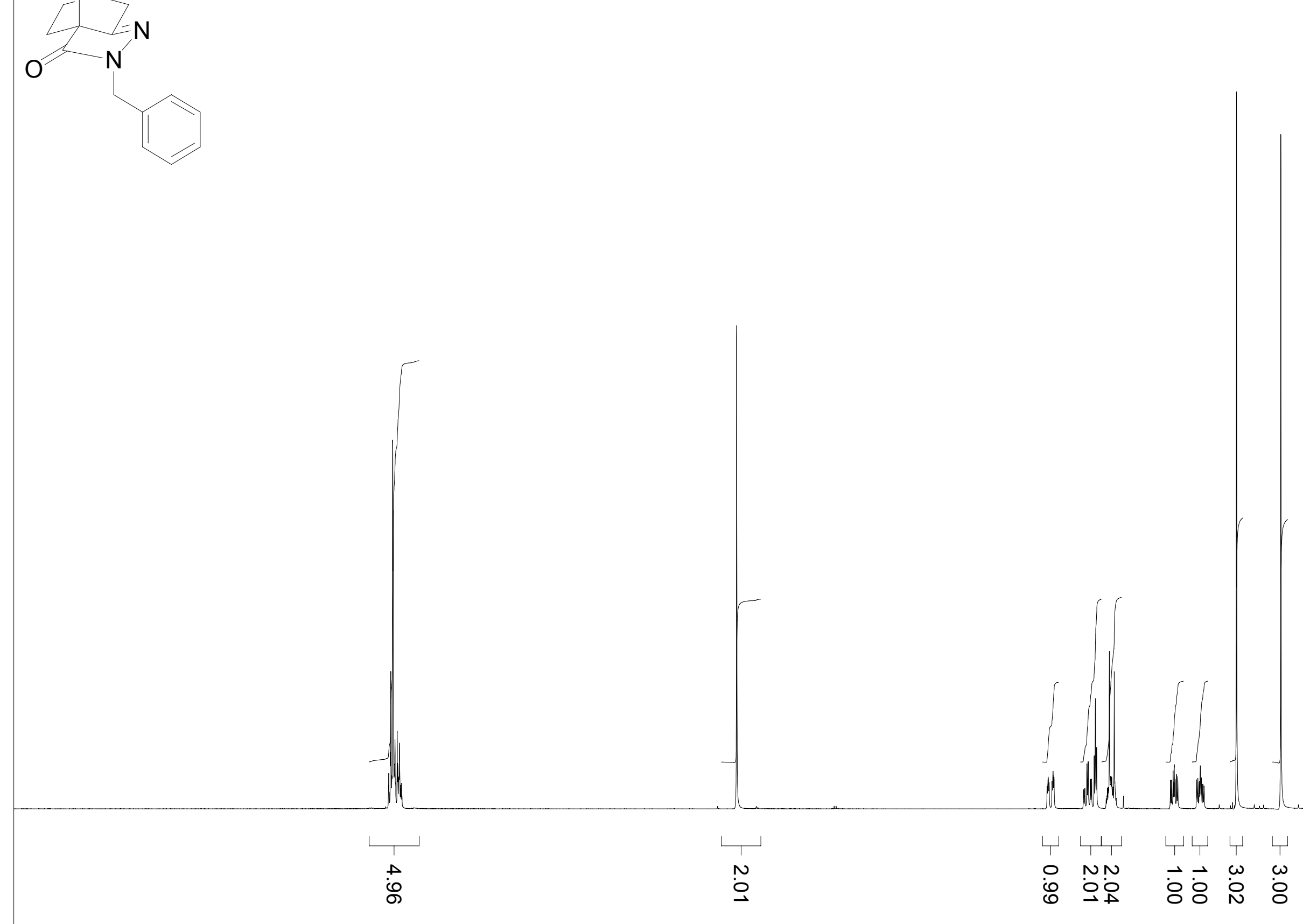

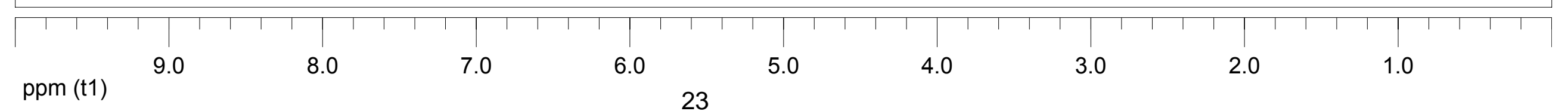




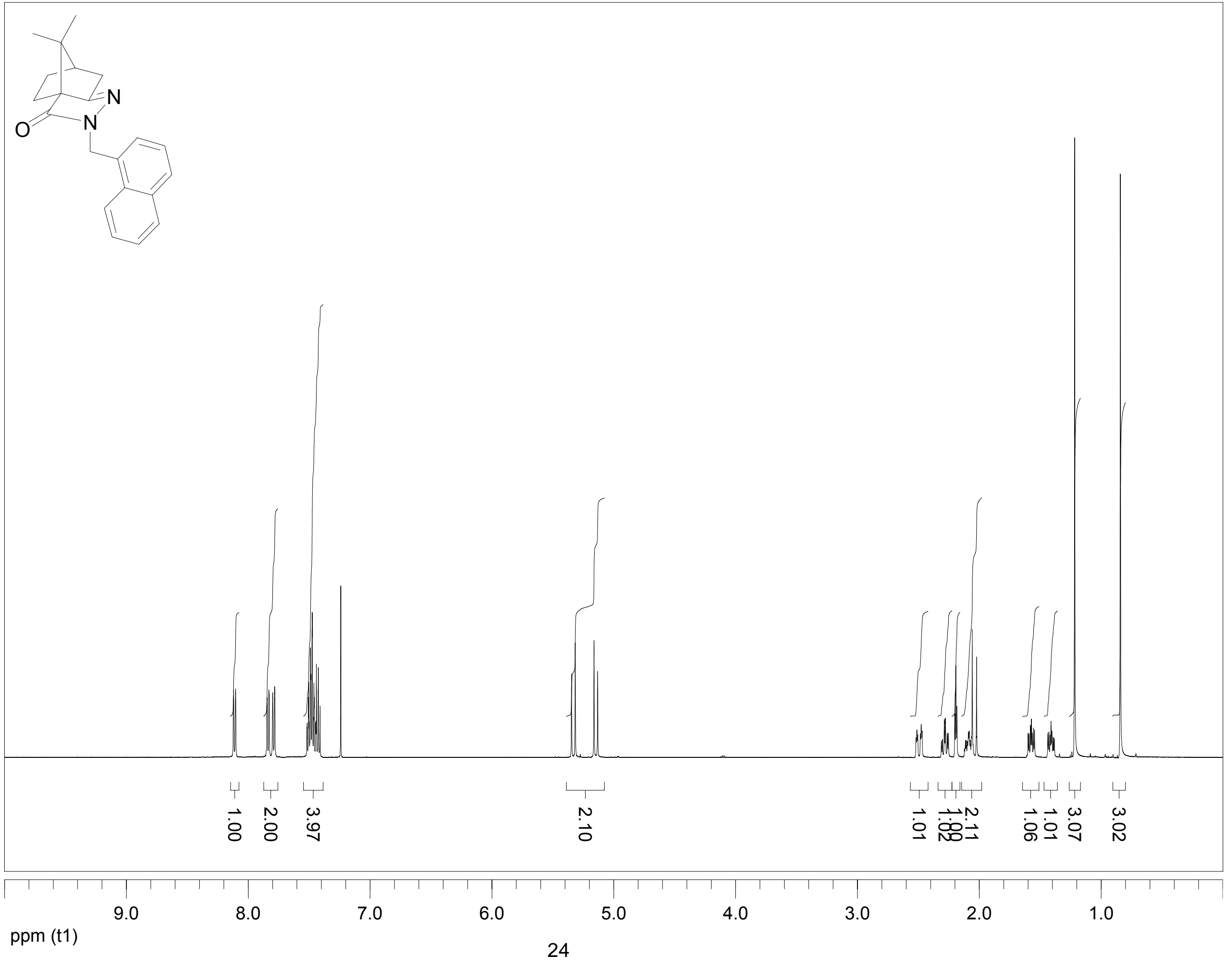


$\mathrm{O} \quad \mathrm{H}$

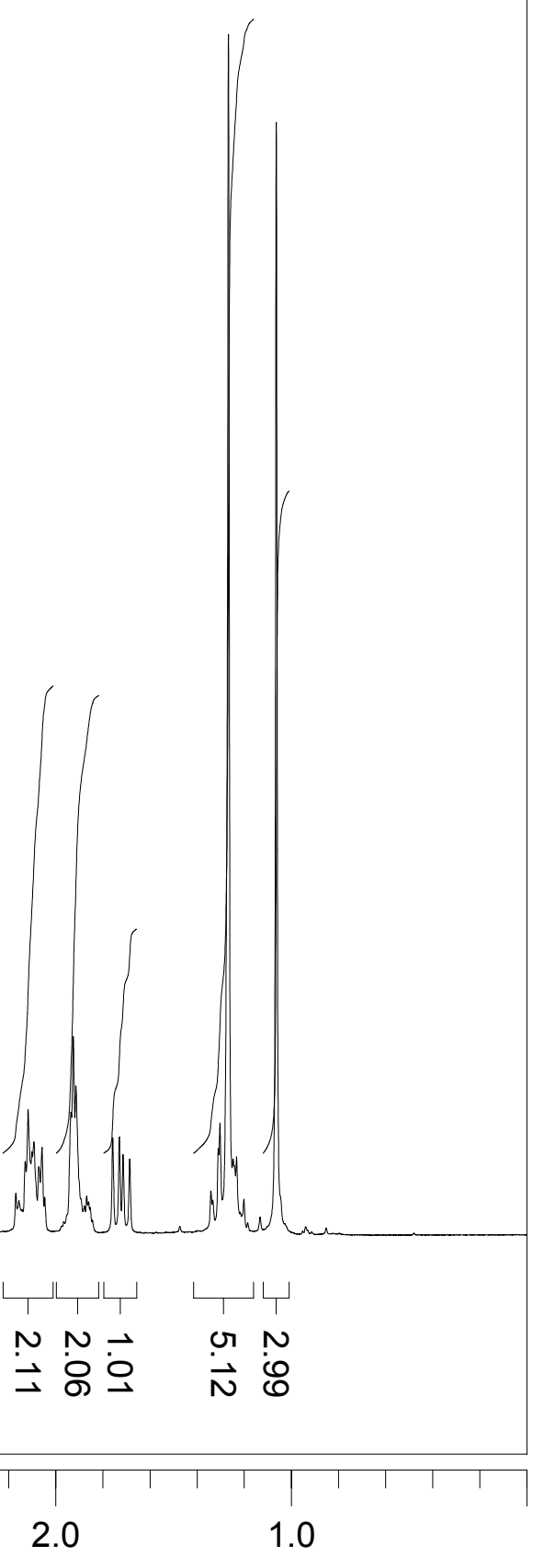

ppm (t1) 
$0-\frac{\mathrm{N}}{\mathrm{CH}_{3}}$ Nullo

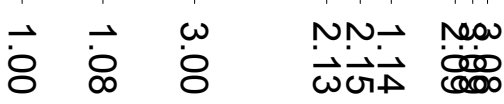




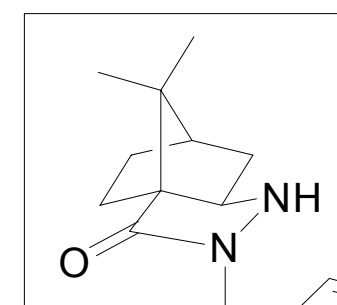




\section{$\mathrm{CHO}$}

$\mathrm{NO}_{2}$

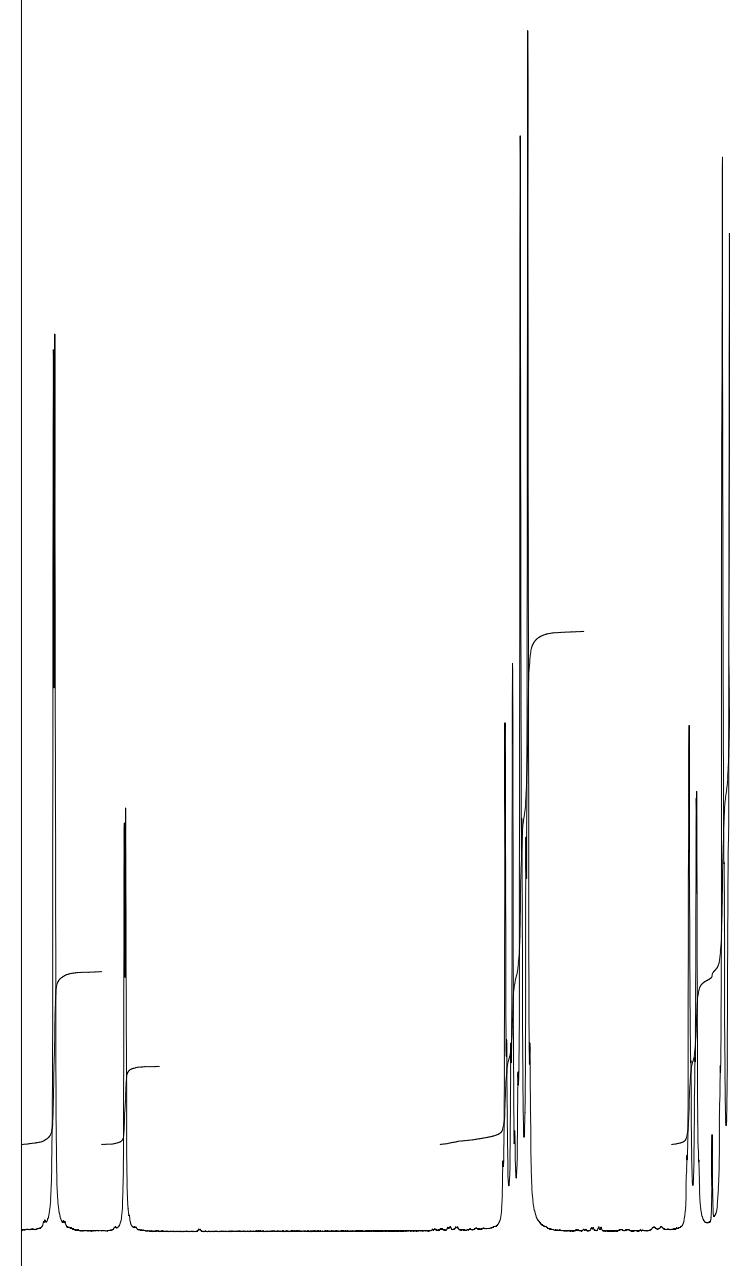

$\overrightarrow{\text { bे }}$

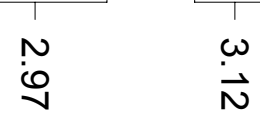

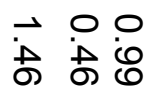

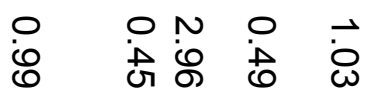

$\stackrel{n}{\dot{n}}$ 

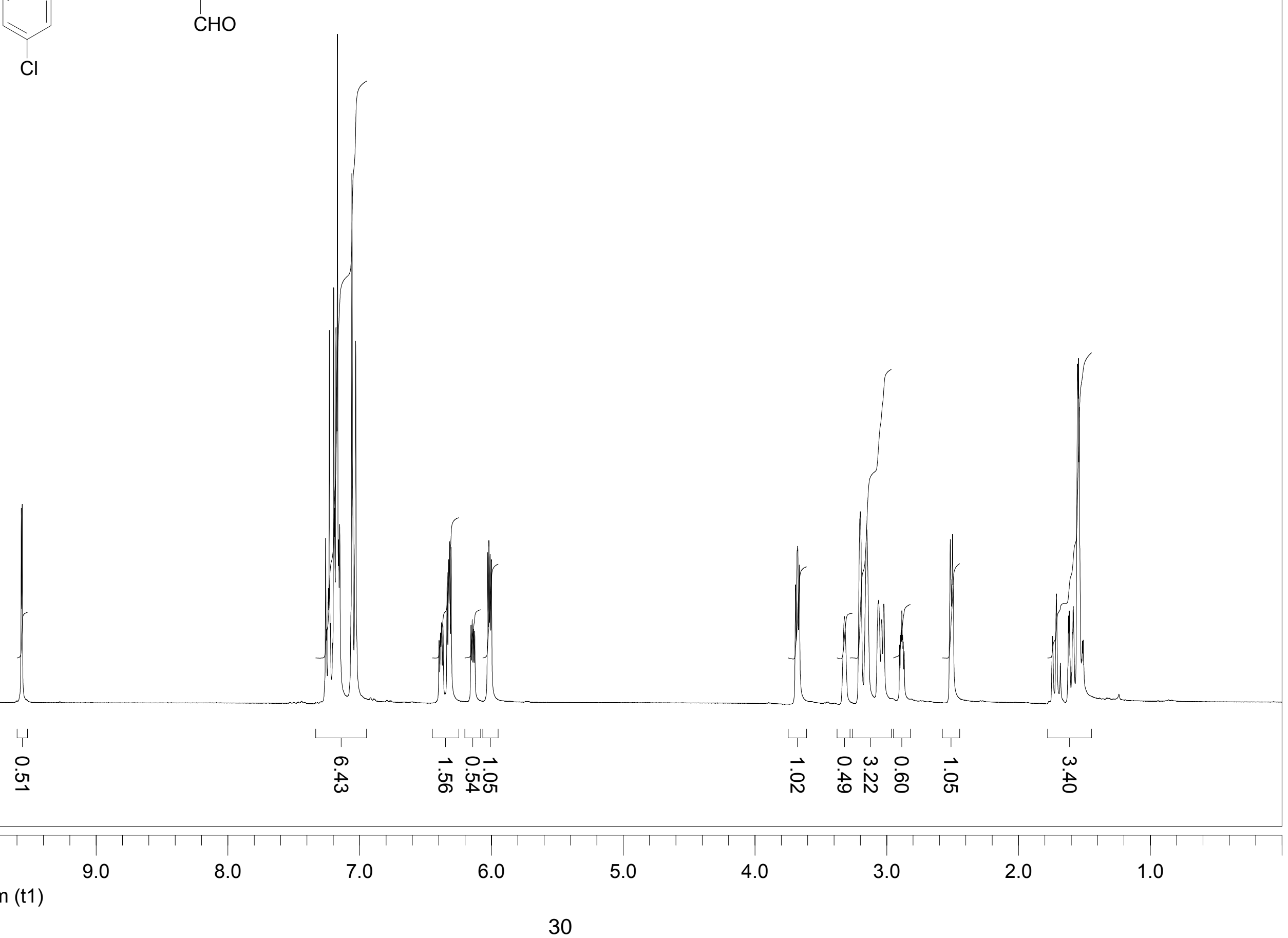


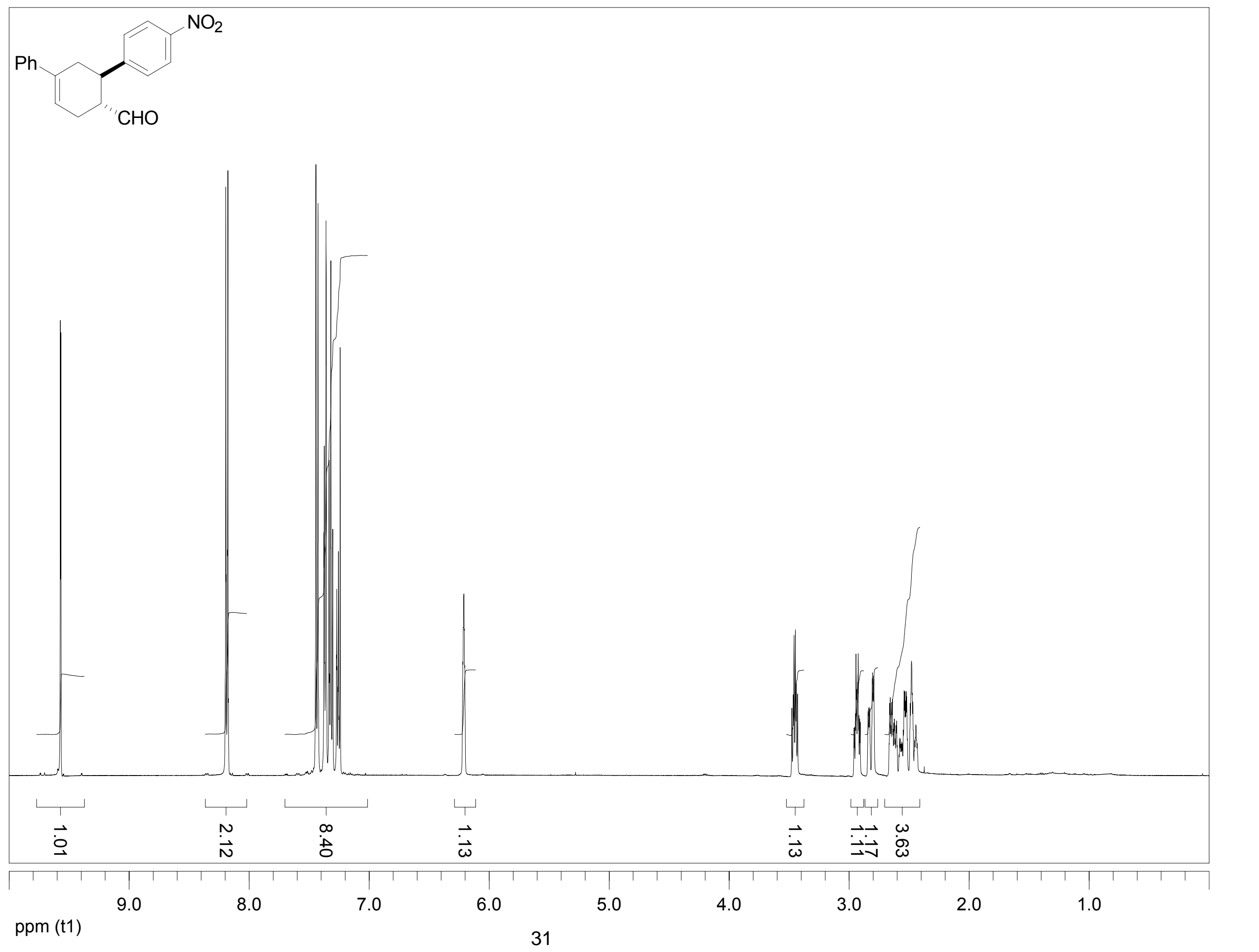




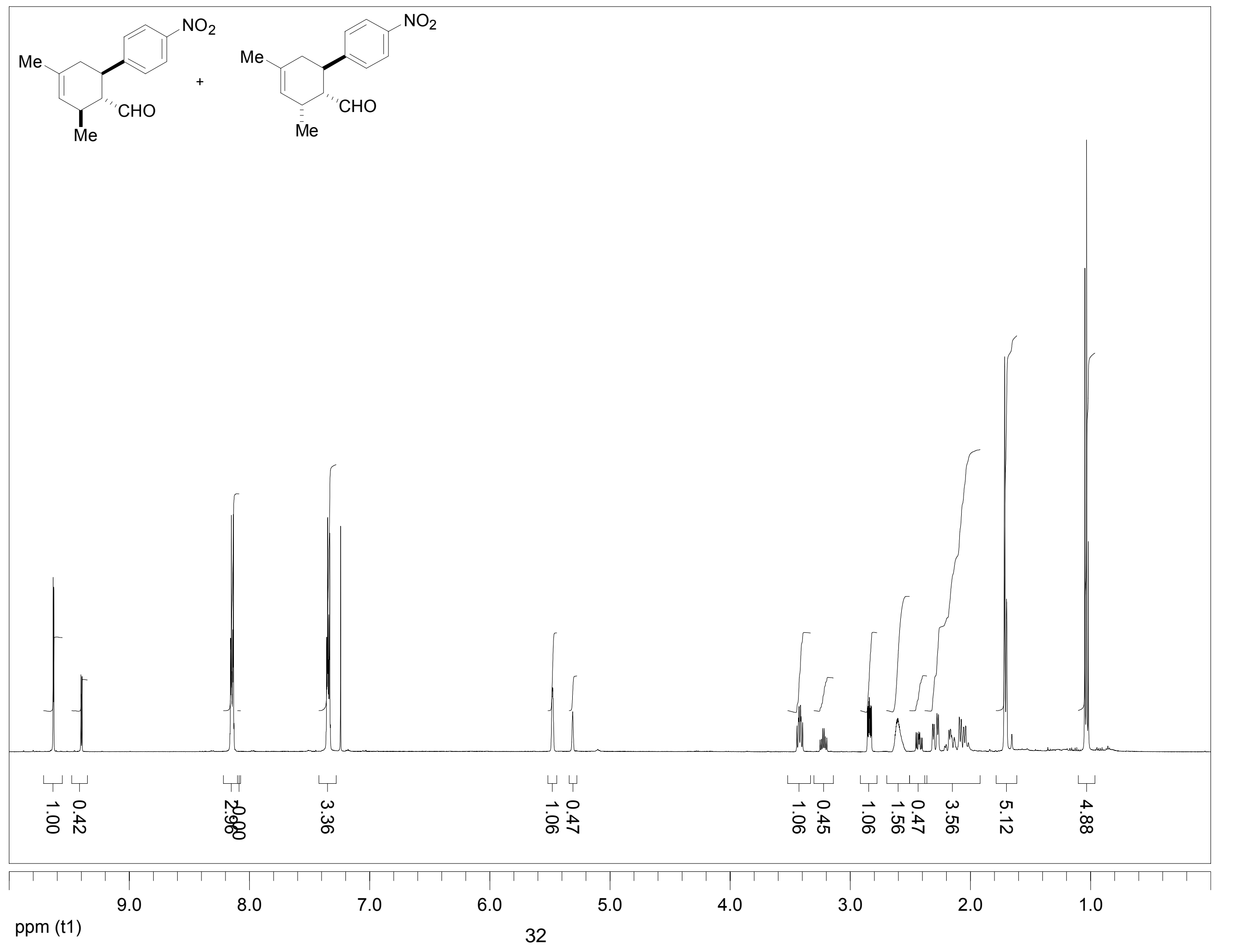



$\mathrm{CHO}$

$\mathrm{NO}_{2}$

$\mathrm{NO}_{2} \quad \mathrm{CHO}$

$\mid$ 